\title{
Implementation of the frequency-modulated sideband search method for gravitational waves from low mass X-ray binaries
}

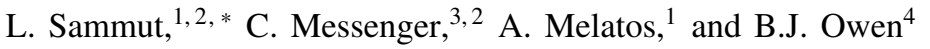 \\ ${ }^{1}$ University Of Melbourne, Victoria 3052, Australia \\ ${ }^{2}$ Albert Einstein Institute, Callinstraße 38,D-30167 Hannover,Germany \\ ${ }^{3}$ University of Glasgow, Glasgow, G12 8QQ, United Kingdom \\ ${ }^{4}$ The Pennsylvania State University, University Park, Pennsylvania 16802, USA \\ (Dated: August 21, 2018) \\ (commitID: 1d41f0b94a6df042af7e99a97529058a81632422) \\ (commitDate: Wed Nov 6 12:08:06 2013 +0000)
}

(LIGO-P1300118)

\begin{abstract}
We describe the practical implementation of the sideband search, a search for periodic gravitational waves from neutron stars in binary systems. The orbital motion of the source in its binary system causes frequencymodulation in the combination of matched filters known as the $\mathcal{F}$-statistic. The sideband search is based on the incoherent summation of these frequency-modulated $\mathcal{F}$-statistic sidebands. It provides a new detection statistic for sources in binary systems, called the $C$-statistic. The search is well suited to low-mass X-ray binaries, the brightest of which, called Sco X-1, is an ideal target candidate. For sources like Sco X-1, with well constrained orbital parameters, a slight variation on the search is possible. The extra orbital information can be used to approximately demodulate the data from the binary orbital motion in the coherent stage, before incoherently summing the now reduced number of sidebands. We investigate this approach and show that it improves the sensitivity of the standard Sco X-1 directed sideband search. Prior information on the neutron star inclination and gravitational wave polarization can also be used to improve upper limit sensitivity. We estimate the sensitivity of a Sco X-1 directed sideband search on 10 days of LIGO data and show that it can beat previous upper limits in current LIGO data, with a possibility of constraining theoretical upper limits using future advanced instruments.
\end{abstract}

PACS numbers: 95.85.Sz, 97.60.Jd, 97.80.Jp

\section{INTRODUCTION}

Rotating neutron stars are the main candidates for sources of persistent, periodic gravitational radiation detectable by ground-based, long-baseline gravitational wave interferometers. Time varying quadrupole moments in (and thus gravitational-wave emission from) these sources can result from deformations of the solid crust (and possibly a solid core) supported by elastic stresses [1-6], deformations of various parts of the star supported by magnetic stresses [7- 12], or free precession [13, 14] or long-lived oscillation modes of the entire star [15. 19$]$.

Neutron stars in accreting binary systems are an important sub-class of periodic gravitational wave sources. Accretion may trigger or enhance the aforementioned gravitational-wave emission mechanisms, creating or driving the quadrupole moment toward its maximum value through thermal, magnetic, or other effects [1, 16, 20,24]. If a balance is assumed between the gravitational radiation-reaction torque and the accretion torque [1, 25, 26], then the strongest emitters of continuous gravitational waves are predicted to be sources in low-mass X-ray binaries (LMXBs), specifically those accreting at the highest rate [27, 28].

Given the estimated ages $\left(\sim 10^{10} \mathrm{yrs}\right)$ and observed accretion rates of LMXBs (reaching near the Eddington limit of

*Electronic address: 1sammut@ student.unimelb.edu.au
$\left.\dot{M}_{\text {Edd }}=2 \times 10^{-8} M_{\odot} \mathrm{yr}^{-1}\right)$, accretion is expected to spin-up the neutron star beyond the breakup frequency $(\sim 1.5 \mathrm{kHz}$ for standard neutron star equations-of-state [29, 30]). However, measured spin frequencies of LMXB neutron stars (from X-ray pulsations or thermonuclear bursts) so far range only from 95 to $619 \mathrm{~Hz}$ [28, 31-33]. The spin frequency cut-off lies well below breakup, and suggests the existence of a spin-down torque to balance the spin-up from accretion. A possible explanation, proposed by Papaloizou and Pringle [25] and advanced by Wagoner [26] and Bildsten [1], is gravitational radiation. The torque-balance scenario implies a relation between the X-ray flux, spin frequency and gravitational wave strain; the more luminous the $\mathrm{X}$-ray source, the greater the strain. Scorpius $\mathrm{X}-1$ (Sco X-1), the brightest LMXB, is therefore a promising target for periodic gravitational wave searches.

The global network of kilometer-scale, Michelson-type laser interferometers are sensitive to gravitational waves in the $O(10-1000) \mathrm{Hz}$ frequency band. The Laser Interferometer Gravitational-Wave Observatory (LIGO) detectors achieved design sensitivity during the fifth science run (S5), between November 2005 and October 2007 [34, 35]. LIGO consists of three Michelson interferometers (one with $4 \mathrm{~km}$ arms at Livingston, Louisiana, and two co-located at Hanford, Washington, with $4 \mathrm{~km}$ and $2 \mathrm{~km}$ arms) separated by $\sim 3000 \mathrm{~km}$. Together the LIGO [36], Virgo [37, 38] and GEO600 [39, 40] detectors form a world-wide network of broad-band interferometric gravitational wave observatories in an international effort to directly detect gravitational wave emission for the first time.

The LIGO Scientific Collaboration has so far published 
three main types of searches for periodic or continuous gravitational wave emission; targeted, directed and all-sky searches. Targeted searches are the most sensitive since they have the most tightly constrained parameter space. They target known sources, such as radio pulsars, with very wellconstrained sky position, spin frequency and frequency evolution, and binary parameters (if any). These searches are fully-coherent, requiring accurately known prior phase information, making them computationally expensive to perform over large regions of parameter space [41-43]. Targeted LIGO and Virgo searches have already set astrophysically interesting upper limits (e.g. beating theoretical indirect limits) on some pulsar parameters such as the gravitational wave strain from the Crab pulsar [43, 44] and the Vela pulsar [45]. Directed searches aim at a particular sky location but search for unknown frequency (and/or frequency evolution). In most cases so far, directed searches have used a fully-coherent approach and approached the limits of computational feasibility. The search directed at the (possible) neutron star in the direction of the supernova remnant Cassiopeia A was able to beat indirect limits [46]. The third type of continuous gravitational wave search, the wide parameter-space searches, are also computationally intensive. They can involve searching over the entire sky or any comparably large parameter space, and usually employ semi-coherent approaches, combining short coherently analyzed segments in an incoherent manner. This process is tuned to balance the trade-off between reduced computational load and reduced sensitivity. The all-sky searches presented in [47-51] target isolated neutron star sources (i.e. those not in binary systems). There is also an all-sky search for neutron stars in binary systems currently being run on LIGO's latest S6 data run [52].

Directed searches can also be made for known accreting neutron stars in binaries, and LIGO has previously conducted two of these searches for Sco X-1. The first, a coherent analysis using data from the second science run (S2), was computationally limited to the analysis of six-hour data segments from the LIGO interferometers, and placed $95 \%$ upper limits on the wave strain of $h_{0}^{95 \%} \approx 2 \times 10^{-22}$ for two different $20 \mathrm{~Hz}$ bands [53]. This search utilized a maximum likelihood detection statistic based on matched filtering called the $\mathcal{F}$-statistic [54]. The second, a directed version of the all-sky, stochastic, crosscorrelation analysis, known as the "Radiometer" search, was first conducted on all 20 days of data from the $\mathrm{S} 4$ science run [55], and later on the $\sim 2$ year S5 data reporting a 90\% upper limit on the root-mean-square (RMS) strain $h_{\mathrm{rms}}^{90 \%}>5 \times 10^{-25}$ (over the range $40-1500 \mathrm{~Hz}$, with the minimum around 150 $\mathrm{Hz}$ [56].

Semi-coherent search methods provide a compromise between sensitivity and the computational cost of a fully coherent search. They should be the most sensitive at fixed computing cost [57, 58]. A fast and robust search strategy for the detection of signals from binary systems in gravitational wave data was proposed in [59]. The signal from a source in a binary system is phase- (or frequency-) modulated due its periodic orbital motion, forming "sidebands" in the gravitational wave frequency spectrum. In searching detector data, this technique, called the sideband search, uses the same co- herent $(\mathcal{F}$-statistic) stage as the previous coherent (S2) search. It then combines the frequency-modulated sidebands arising in $\mathcal{F}$-statistic data in a (computationally inexpensive) incoherent stage, reducing the need for a large template bank. This approach is based on a method that has successfully been employed in searches for binary pulsars in radio data [60].

Here we develop this sideband technique into a search pipeline and present a detailed description of how it is applied to gravitational wave detector data, as well as the expected sensitivity. The paper is set out as follows. Section [I] briefly describes the astrophysics of LMXBs and their predicted gravitational wave signature. The search algorithm is described in detail in Section III Section IV] outlines the parameter space of the search allowing primary sources to be identified in Section $\mathrm{V}$. The statistical analysis of the results of the search is described in Section VI. along with a definition of the upper limits of the search. The sensitivity of the search is discussed in Section VII A brief summary is provided in Section VIII with a discussion of the limitations and future prospects of the search.

\section{LOW MASS X-RAY BINARIES}

LMXBs are stellar systems where a low-magnetic-field ( $\lesssim 10^{9} \mathrm{G}$ ) compact object (primary) accretes matter from a lower-mass (secondary) companion $\left(<1 M_{\odot}\right)$ [27, 61]. The compact objects in LMXB systems can be black holes, neutron stars or white dwarfs. For gravitational wave emission we are interested in LMXBs with neutron stars as the primary mass (typically $\sim 1.4 M_{\odot}$ ), since neutron stars can sustain the largest quadrupole moment.

Observations of thermal X-ray emission from the inner region of the accretion disk provide a measurement of the accretion rates in LMXBs. The range of observed accretions rates is broad, ranging from $10^{-11} M_{\odot} \mathrm{yr}^{-1}$ to the Eddington limit, $\dot{M}_{\text {Edd }}=2 \times 10^{-8} M_{\odot} \mathrm{yr}^{-1}$ [30]. Some LMXBs also exhibit periodic pulsations or burst type behavior, and so provide a means of measuring the spin frequency $v_{\mathrm{s}}$ of the neutron star in the system. The measured $v_{\mathrm{s}}$ of these systems lie in the range of $95 \leq v_{\mathrm{s}} \leq 619 \mathrm{~Hz}$ [31-33]. The broad range of accretion rates coupled with the estimated age of these systems $\left(\sim 10^{10}\right.$ years implied by evolutionary models [30, 62]) would suggest a greater upper limit on observed spin frequency since accretion exerts substantial torque on the neutron star. However, none of these systems have yet been observed to spin at or near the breakup frequency $v_{b} \sim 1.5 \mathrm{kHz}\left(v_{b} \gtrsim 1 \mathrm{kHz}\right.$ for most equations of state [29, 30]). The maximum observed spin frequency falls far below the theorized breakup frequency and suggests a competing (damping) mechanism to the spinup caused by accretion. One explanation for the observed spin frequency distribution of LMXBs is that the spin-up from the accretion torque is balanced by a gravitational wave spindown torque [1, 26]. Since the gravitational wave spin-down torque scales like $v_{\mathrm{s}}^{5}$ (see Eq. 3 below), a wide range of accretion rates then leads to a rather narrow range of equilibrium rotation rates, as observed. 


\section{A. Gravitational wave emission}

Using the torque balancing argument from Wagoner [26] and Bildsten [1], we can estimate the gravitational wave strain amplitude emitted from accreting binary systems from their observable X-ray flux. This is a conservative upper limit as it assumes all angular momentum gained from accretion is completely converted into gravitational radiation.

The intrinsic strain amplitude $h_{0}$ for a system with angular spin frequency $\Omega_{\mathrm{s}}=2 \pi v_{\mathrm{s}}$ at a distance $d$ from an observer emitting gravitational waves via a mass quadrupole can be expressed as

$$
h_{0}=\frac{4 G Q}{c^{4} d} \Omega_{\mathrm{s}}^{2},
$$

where $G$ is the gravitational constant, $c$ is the speed of light and the quadrupole moment $Q=\epsilon I$ is a function of the ellipticity $\epsilon$ and moment of inertia $I$ [54]. This can be expressed in terms of the spin-down (damping) torque $N_{\mathrm{GW}}$ due to gravitational radiation giving

$$
h_{0}^{2}=\frac{5 G}{8 c^{3} d^{2} \Omega_{\mathrm{s}}^{2}} N_{\mathrm{GW}},
$$

where

$$
N_{\text {GW }}=\frac{32 G Q^{2}}{5 c^{5}} \Omega_{\mathrm{s}}^{5} .
$$

The accretion torque $N_{\mathrm{a}}$ applied to a neutron star of mass $M$ and radius $R$ accreting at a rate $\dot{M}$ is given by

$$
N_{\mathrm{a}}=\dot{M} \sqrt{G M R}
$$

Assuming that the X-ray luminosity can be written as $L_{\mathrm{X}}=$ $G M \dot{M} / R$, the accretion rate $\dot{M}$ can be expressed as a function of the X-ray flux $F_{\mathrm{X}}$, such that

$$
\dot{M}=\frac{4 \pi R d^{2}}{G M} F_{\mathrm{X}},
$$

since $L_{\mathrm{x}}=4 \pi d^{2} F_{\mathrm{x}}$. In equilibrium, where gravitational radiation balances accretion torque, $N_{\mathrm{GW}}=N_{\mathrm{a}}$. The square of the gravitational wave strain from Eq. 6 can then be expressed in terms of the observable X-ray flux such that

$$
h_{0}^{2}=\frac{5}{3} \frac{F_{\mathrm{X}}}{v_{\mathrm{S}}}\left(\frac{G R^{3}}{M}\right)^{1 / 2} .
$$

Selecting fiducial values for the neutron star mass, radius, Xray flux, and spin frequency (around the middle of the observed range), we can express the equilibrium strain upper limit $h_{0}^{\mathrm{EQ}}$ in terms of $v_{\mathrm{s}}$ and $F_{\mathrm{X}}$ via

$h_{0}^{\mathrm{EQ}}=5.5 \times 10^{-27}\left(\frac{F_{\mathrm{x}}}{F_{*}}\right)^{1 / 2}\left(\frac{R}{10 \mathrm{~km}}\right)^{3 / 4}\left(\frac{1.4 M_{\odot}}{M}\right)^{1 / 4}\left(\frac{300 \mathrm{~Hz}}{v_{\mathrm{s}}}\right)^{1 / 2}$,

(7)

where $F_{*}=10^{-8} \mathrm{erg} \mathrm{cm}^{-2} \mathrm{~s}^{-1}$. If the system emits gravitational waves via current quadrupole radiation instead, as is the case with $r$-mode oscillations, the relation between gravitational wave frequency and spin frequency differs. In this case the preceding equations are modified slightly, requiring roughly $v_{\mathrm{s}} \rightarrow(2 / 3) v_{\mathrm{s}}[63]$. However these expressions, and the rest of the analysis except where otherwise noted, do not change if expressed in terms of the gravitational-wave frequency.

The resulting relation in Eq. 7 implies that LMXBs that accrete close to the Eddington limit are potentially strong gravitational wave emitters. Of these potentially strong sources, Sco X-1 is the most promising due to its observed X-ray flux [28].

\section{SEARCH ALGORITHM}

In this section we define our detection statistic and show how it exploits the characteristic frequency-modulation pattern inherent to sources in binary systems.

Fully-coherent, matched-filter searches for continuous gravitational waves can be described as a procedure that maximizes the likelihood function over a parameter space. The amplitude parameters (gravitational wave strain amplitude $h_{0}$, inclination $\iota$, polarization $\psi$ and reference phase $\phi_{0}$ ) can, in general, be analytically maximized, reducing the dimensions of this parameter space [54]. These parameters define the signal amplitudes in our signal model. Analytic maximization leaves the phase-evolution parameters (gravitational wave frequency $f_{o}$ and its derivatives $f^{(k)}$ and sky position $\left.[\alpha, \delta]\right)$ to be numerically maximized over. Numerical maximization is accomplished through a scheme of repeated matched-filtering performed over a template bank of trial waveforms defined by specific locations in the phase parameter space, which is typically highly computationally expensive [28, 41, 53].

The method we outline here makes use of the fact that we know the sky position of our potential sources and, hence, the phase evolution due to the motion of the detector can be accurately accounted for. We also know that the phase evolution due to the binary motion of the source will result in a specific distribution of signal power in the frequency domain. This distribution has the characteristics that signal power is divided amongst a finite set of frequency-modulation sidebands. The number of sidebands and their relative frequency spacing can be predicted with some knowledge of the binary orbital parameters.

In order to avoid the computational limits imposed by a fully-coherent parameter space search, we propose a single fully-coherent analysis stage, that accounts for detector motion only, is followed by a single incoherent stage in which the signal power contained within the frequency-modulated sidebands in summed to form a new detection statistic. This summing procedure is accomplished via the convolution of an approximate frequency domain power template with the output of the coherent stage.

The three main stages of the search, the $\mathcal{F}$-statistic, sideband template, and $C$-statistic, are graphically illustrated in Figure 1 1 In this noise-free example, the frequencymodulation sidebands are clearly visible. The $\mathcal{F}$-statistic 
is also amplitude-modulated due to the daily variation of the detector antenna response, resulting in the amplitudemodulation applied to each frequency-modulation sideband. The second panel represents the approximate frequency domain template, a flat comb function with unit amplitude teeth (the spikes or delta functions). When convolved with the $\mathcal{F}$-statistic in the frequency domain we obtain the $C$-statistic shown in the right-hand panel. The maximum power is clearly recovered at the simulated source frequency.

The following sub-sections discuss each of the search components in more detail. Section III A presents the phase model used to characterize the gravitational wave signal from a source in a binary system. The signal model is introduced in Sec. III B. The $\mathcal{F}$-statistic is introduced in Section III C and its behavior as a function of search frequency is described in Sec. IIID Section IIIE then goes on to describe how matching a filter for an isolated neutron star system to a signal from a source in a binary system results in frequency-modulated sidebands appearing in the output of the $\mathcal{F}$-statistic. The detection statistic for gravitational wave sources in binary orbits is fully described as the incoherent sum of frequency-modulated $\mathcal{F}$-statistics in Sec. IIIF The simplest, unit amplitude, sideband template, and its justification over a more realistic template, are discussed in Sec. III G. A more sensitive implementation, incorporating an approximate binary phase model in the calculation of the $\mathcal{F}$-statistic and reducing the width of the frequency-modulated sideband pattern by the fractional errors on the semi-major axis parameters, is discussed in Sec. III H. Beginning in this section and continuing in the following sections, we have made a distinction between the intrinsic values of a search parameter $\theta_{0}$ (denoted with a subscript zero) and the observed values $\theta$ (no subscripts).

\section{A. Phase model}

The phase of the signal at the source can be modeled by a Taylor series such that

$$
\Phi(\tau)=2 \pi \sum_{k=0}^{s} \frac{f^{(k)}}{(k+1) !}\left(\tau-\tau_{0}\right)^{k+1}
$$

where $f^{(k)}$ represents the $k^{\text {th }}$ time derivative of the gravitational wave frequency evaluated at reference time $\tau_{0}$. For the purposes of this work we restrict ourselves to a monochromatic signal and hence set $f^{(k)}=0$ for all $k>0$ and define $f_{0}=\left.f^{(0)}\right|_{\tau_{0}}=f\left(\tau_{0}\right)$ as the intrinsic frequency. We discuss this choice in Sec. IVB. The phase received at the detector is $\Phi\left[\tau\left(t\left(t^{\prime}\right)\right)\right]$, where we define the retarded times measured at the Solar system barycenter (SSB) and detector as $t$ and $t^{\prime}$ respectively. The relation between $t$ and $t^{\prime}$ is a function of source sky position relative to detector location and, since we only concern ourselves with sources of known sky position, we assume that the effects of phase contributions from detector motion can be exactly accounted for. For this reason we work directly within the SSB frame. The relationship between the source and retarded times for a non-relativistic eccentric binary orbit is given by [64]

$$
t=\tau+a_{0}\left[\sqrt{1-e^{2}} \cos \omega \sin E+\sin \omega(\cos E-e)\right],
$$

where $a_{0}$ is the light crossing time of the semi-major axis projected along the line of sight. The orbital eccentricity is defined by $e$ and the argument of periapse, given by $\omega$, is the angle in the orbital plane from the ascending node to the direction of periapse. The variable $E$ is the eccentric anomaly defined by $2 \pi\left(\tau-t_{p}\right) / P=E-e \sin E$, where $t_{p}$ is the time of passage through periapse (the point in the orbit where the two bodies are at their closest) and $P$ is the orbital period.

It is expected that dissipative processes within LMXBs drive the orbits to near circularity. In the low eccentricity limit $e \ll 1$, we obtain the following expression

$$
\begin{aligned}
t=\tau & +a_{0}\left\{\sin \left[\Omega\left(\tau-t_{a}\right)\right]+\frac{e \cos \omega}{2} \sin \left[2 \Omega\left(\tau-t_{p}\right)\right]\right. \\
& \left.\left.+\frac{e \sin \omega}{2} \cos \left[2 \Omega\left(\tau-t_{p}\right)\right]\right\}+O\left(e^{2}\right)\right\},
\end{aligned}
$$

where $\Omega=2 \pi / P$ and we have used the time of passage through the ascending node $t_{a}=t_{p}-\omega / \Omega$ as our reference phase in the first term. For circular orbits, where $e=0$, the expression reduces to only this first term. Using $t_{a}$ is sensible in this case since $t_{p}$ and $\omega$, are not defined in a circular orbit. Note that the additional eccentric terms are periodic at multiples of twice the orbital frequency. Using Eq. 10, we would expect to accumulate timing errors of order $\mu$ s for the most eccentric known LMXB systems. We shall return to this feature in Sec. IVE.

To write the gravitational wave phase as a function of SSB time, we invert Eq. 10 to obtain $\tau(t)$. The binary phase can be corrected for in a standard matched filter approach, where Eq.9 is solved numerically. In our method we instead approximate the inversion as

$$
\Phi(t) \simeq 2 \pi f_{0}\left(t-t_{0}\right)-2 \pi f_{0} a_{0} \sin \left[\Omega\left(t-t_{a}\right)\right]
$$

for circular orbits. ${ }^{1}$ Under this approximation the signal phase can be represented as a linear combination of the phase contributions from the spin of the neutron star $\phi_{\text {spin }}$ and from the binary orbital motion of the source $\phi_{\text {bin }}$, such that

$$
\begin{aligned}
\phi_{\text {spin }} & =2 \pi f_{0}\left(t-t_{0}\right) \\
\phi_{\text {bin }} & =-2 \pi f_{0} a_{0} \sin \left[\Omega\left(t-t_{a}\right)\right] .
\end{aligned}
$$

\section{B. Signal model}

We model the data $\boldsymbol{x}(t)$ collected by a detector located at the SSB as the signal $\boldsymbol{s}(t)$ plus stationary Gaussian noise $\boldsymbol{n}(t)$ so that

$$
\boldsymbol{x}(t)=\boldsymbol{s}(t)+\boldsymbol{n}(t)
$$

\footnotetext{
${ }^{1}$ Phase errors caused by this inversion approximation amount to maximum phase offsets of $\sim 2 \pi f_{0} a_{0}^{2} \Omega$.
} 


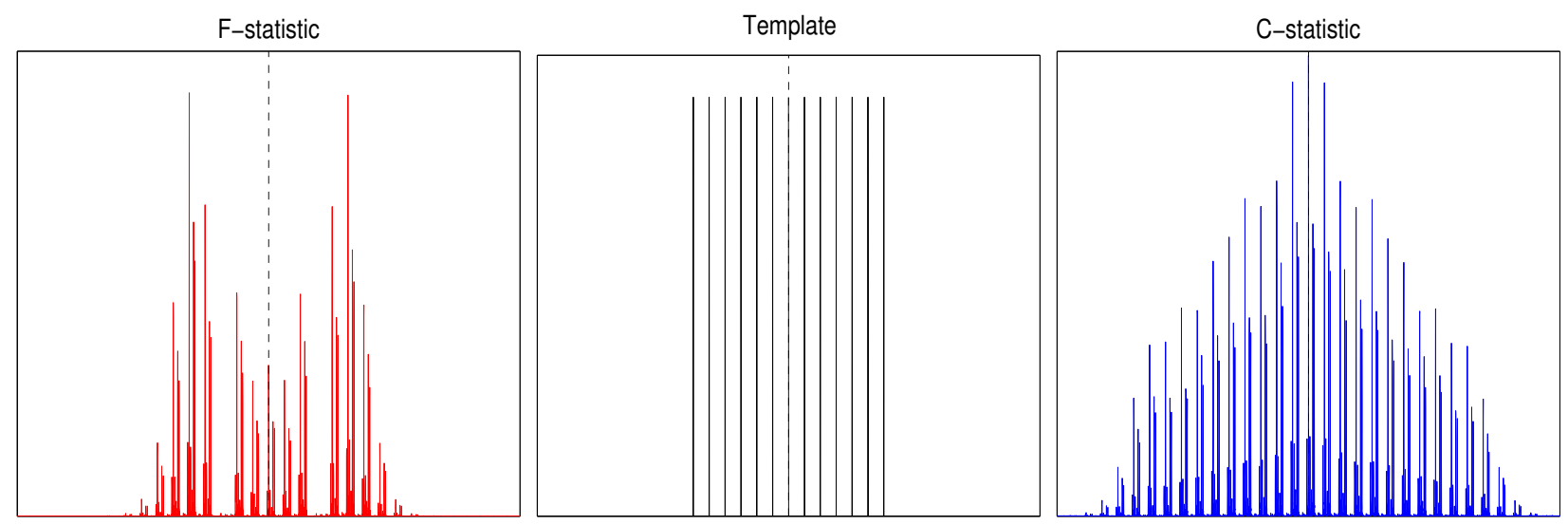

FIG. 1: Graphical illustration of the sideband search pipeline, showing the frequency-modulated $\mathcal{F}$-statistic (left, red), the sideband template (middle, black), and their convolution, known as the $C$-statistic (right, blue). In this noise free case, a signal of $f_{0}=200 \mathrm{~Hz}$ with amplitude $h_{0}=1 \mathrm{in}$ a system with $a=0.005 \mathrm{~s}, P=7912.7 \mathrm{~s}$, sky position $\alpha=2.0 \mathrm{rad}, \delta=1.0 \mathrm{rad}$, and phase parameters $\psi=0.2 \mathrm{rad}, \cos \iota=0.5, \phi_{0}=1$ $\mathrm{rad}$, was simulated for a 10 day observation span. Frequency increases along the horizontal axis, which ranges from 199.998 to $200.002 \mathrm{~Hz}$ on each plot. In each case the location of the injected signal at $200 \mathrm{~Hz}$ is indicated by the vertical dashed black line.

with

$$
\boldsymbol{s}(t)=\mathcal{A}_{\mu} \boldsymbol{h}_{\mu}(t),
$$

where we employ the Einstein summation convention for $\mu=1,2,3,4$. The coefficients $\mathcal{A}_{\mu}$ are independent of time, detector location and orientation. They depend only on the signal amplitude parameters $\lambda=\left\{h_{0}, \psi, \iota, \phi_{0}\right\}$, where $h_{0}$ is the dimensionless gravitational wave strain amplitude, $\psi$ is the gravitational wave polarization angle, $\iota$ is the source inclination angle and $\phi_{0}$ is the signal phase at a fiducial reference time. The coefficients $\mathcal{A}_{\mu}$ are defined as

$$
\begin{aligned}
& \mathcal{A}_{1}=A_{+} \cos \phi_{0} \cos 2 \psi-A_{\times} \sin \phi_{0} \sin 2 \psi \\
& \mathcal{A}_{2}=A_{+} \cos \phi_{0} \sin 2 \psi-A_{\times} \sin \phi_{0} \cos 2 \psi \\
& \mathcal{A}_{3}=A_{+} \sin \phi_{0} \cos 2 \psi-A_{\times} \cos \phi_{0} \sin 2 \psi \\
& \mathcal{A}_{4}=A_{+} \sin \phi_{0} \sin 2 \psi-A_{\times} \cos \phi_{0} \cos 2 \psi
\end{aligned}
$$

where

$$
A_{+}=\frac{1}{2} h_{0}\left(1+\cos ^{2} \iota\right), A_{\times}=h_{0} \cos \iota
$$

are the polarization amplitudes. The time dependent signal components $\boldsymbol{h}_{\mu}(t)$ are defined as

$$
\begin{array}{cc}
h_{1}=a(t) \cos \Phi(t), & h_{2}=b(t) \cos \Phi(t), \\
h_{3}=a(t) \sin \Phi(t), & h_{4}=b(t) \sin \Phi(t),
\end{array}
$$

where $\Phi(t)$ is the signal phase at the detector (which we model as located at the SSB) given by $\mathrm{Eq} 11$ and the antenna pattern functions $a(t)$ and $b(t)$ are described by Eqs. (12) and (13) in [54].

\section{C. $\mathcal{F}$-statistic}

The $\mathcal{F}$-statistic is a matched-filter based detection statistic derived via analytic maximization of the likelihood over unknown amplitude parameters [54]. Let us first introduce the multi-detector inner product

$$
(\boldsymbol{x} \mid \boldsymbol{y})=\sum_{X}\left(x_{X} \mid y_{X}\right)=\sum_{X} \frac{2}{S_{X}(f)} \int_{-\infty}^{\infty} w_{X}(t) x_{X}(t) y_{X}(t) d t,
$$

where $X$ indexes each detector and $S_{X}(f)$ is the detector single-sided noise spectral density. We modify the definitions of [54] and [65] to explicitly include gaps in the time-series by introducing the function $w_{X}(t)$ which has value 1 when data is present and 0 otherwise. This also allows us to extend the limits of our time integration to $(-\infty, \infty)$ since the window function will naturally account for the volume and span of data for each detector.

The $\mathcal{F}$-statistic itself is defined as

$$
2 \mathcal{F}=x_{\mu} \mathcal{M}^{\mu v} x_{v},
$$

where $\mathcal{M}^{\mu v}$ form the matrix inverse of $\mathcal{M}_{\mu \nu}$ and we follow the shorthand notation of [65] defining $x_{\mu} \equiv\left(\boldsymbol{x} \mid \boldsymbol{h}_{\mu}\right)$ and $\mathcal{M}_{\mu v} \equiv$ $\left(\boldsymbol{h}_{\mu} \mid \boldsymbol{h}_{v}\right)$. Evaluation of $\mathcal{M}$ leads to a matrix of the form

$$
\mathcal{M}=\frac{1}{2}\left(\begin{array}{ll}
C & 0 \\
0 & C
\end{array}\right), \quad \text { where } C=\left(\begin{array}{ll}
A & C \\
C & B
\end{array}\right)
$$

where the components

$$
A=(a \mid a), B=(b \mid b), C=(a \mid b),
$$

are antenna pattern integrals. For a waveform with exactly known phase evolution $\Phi(t)$ in Gaussian noise, the $\mathcal{F}$-statistic is a random variable distributed according to a non-central $\chi^{2}$ distribution with 4 degrees of freedom. The non-centrality parameter is equal to the optimal signal-to-noise ratio (SNR)

$$
\rho_{0}^{2}=\frac{1}{2}\left[A\left(\mathcal{A}_{1}^{2}+\mathcal{A}_{3}^{2}\right)+B\left(\mathcal{A}_{2}^{2}+\mathcal{A}_{4}^{2}\right)+2 C\left(\mathcal{A}_{1} \mathcal{A}_{2}+\mathcal{A}_{3} \mathcal{A}_{4}\right)\right]
$$


such that the expectation value and variance of $2 \mathcal{F}$ are given by

$$
\begin{aligned}
\mathrm{E}[2 \mathcal{F}] & =4+\rho_{0}^{2} \\
\operatorname{Var}[2 \mathcal{F}] & =8+4 \rho_{0}^{2},
\end{aligned}
$$

respectively. In the case where no signal is present in the data, the distribution becomes a central $\chi^{2}$-distribution with 4 degrees of freedom.

\section{D. $\mathcal{F}$-statistic and mismatched frequency}

In this section we describe the behavior of the $\mathcal{F}$-statistic as a function of search frequency $f$ for a fixed source frequency $f_{0}$. In this case the inner product that defines $x_{\mu}$ becomes

$$
x_{\mu}=\mathcal{A}_{v}\left(\boldsymbol{h}_{v} \mid \boldsymbol{h}_{\mu}{ }^{\prime}\right)+\left(\boldsymbol{n} \mid \boldsymbol{h}_{\mu}{ }^{\prime}\right),
$$

where $\boldsymbol{h}_{v}$ are the components of a signal with frequency $f_{0}$, and $\boldsymbol{h}_{\mu}^{\prime}$ is a function of search frequency $f$. If we focus on the $\mu=\nu=1$ component as an example we find that

$$
\left(\boldsymbol{h}_{1} \mid \boldsymbol{h}_{1}{ }^{\prime}\right) \cong \sum_{X} \frac{2}{S_{X}(f)} \int_{-\infty}^{\infty} w_{X}(t) a_{X}^{2}(t) \cos (2 \pi f t) \cos \left(2 \pi f_{0} t\right) d t
$$

where we note that the product of cosine functions results in an integrand that contains frequencies at $f-f_{0}$ and $f+f_{0}$. Since both $a_{X}(t)$ and $w_{X}(t)$ are functions that evolve on timescales of hours-days we approximate the contribution from the $f+f_{0}$ component as averaging to zero. We are left with

$$
\begin{aligned}
\left(\boldsymbol{h}_{1} \mid \boldsymbol{h}_{1}{ }^{\prime}\right) & \cong \operatorname{Re}\left[\frac{1}{2} \sum_{X} \frac{2}{S_{X}(f)} \int_{0}^{\infty} w_{X}(t) a_{X}^{2}(t) e^{-2 \pi i\left(f-f_{0}\right) t} d t\right] \\
& \cong \frac{1}{2} \sum_{X} \operatorname{Re}\left[A_{X}\left(f-f_{0}\right)\right]
\end{aligned}
$$

where we have defined the result of the complex integral as $A_{X}(f)$. This is the Fourier transform of the antenna pattern functions weighted by the window function and evaluated at $f-f_{0}$. It is equal to the quantity $\left(a_{X} \mid a_{X}\right)$ when its argument is zero.

The quantity $a_{X}^{2}(t)$ (and similarly $b_{X}^{2}(t)$ and $a_{X}(t) b_{X}(t)$ ) are periodic quantities with periods of 12 and 24 hours plus a non-oscillating component. When in a product with a sinusoidal function and integrated over time they will result in discrete amplitude-modulated sidebands with frequencies at $0, \pm 1 / P_{\oplus}, \pm 2 / P_{\oplus}, \pm 3 / P_{\oplus}, \pm 4 / P_{\oplus} \mathrm{Hz}$ where $P_{\oplus}$ represents the orbital period of the Earth (1 sidereal day). We will ignore all but the zero-frequency components of these functions for the remainder of this paper. We do note that complications regarding the overlap of amplitude-modulated and frequencymodulated sidebands (discussed in the next section) will only arise for sources in binary orbits with periods equal to those present in the antenna pattern functions.
In addition, the window function describing the gaps in the data will influence $A_{X}(f)$. For a gap-free observation the window function serves to localize signal power to within a frequency range $\sim 1 / T$ where $T$ is the typical observation length. When gaps are present this range is broadened and has a deterministic shape given by the squared modulus of the Fourier transform of the window function. We can therefore use a further approximation that

$$
A_{X}\left(f-f_{o}\right) \approx\left(a_{X} \mid a_{X}\right) \frac{\tilde{w}_{X}\left(f-f_{o}\right)}{\tilde{w}_{X}(0)}
$$

where the Fourier transform of the window function is normalized by $\tilde{w}_{X}(0) \equiv \int d t w_{X}(t)$ such that it has a value of unity at the true signal frequency.

We now define the antenna-pattern weighted window function as

$$
\begin{aligned}
\tilde{W}\left(f-f_{0}\right) & \cong \sum_{X} \frac{\left(a_{X} \mid a_{X}\right)}{A} \frac{\tilde{w}_{X}\left(f-f_{o}\right)}{\tilde{w}_{X}(0)} \\
& \cong \sum_{X} \frac{\left(b_{X} \mid b_{X}\right)}{B} \frac{\tilde{w}_{X}\left(f-f_{o}\right)}{\tilde{w}_{X}(0)} \\
& \cong \sum_{X} \frac{\left(a_{X} \mid b_{X}\right)}{C} \frac{\tilde{w}_{X}\left(f-f_{o}\right)}{\tilde{w}_{X}(0)},
\end{aligned}
$$

which is true for observation times $T_{X} \gg$ days. This complex window function has the property that $\tilde{W}(0)$ is a real quantity with maximum absolute value of unity when the template frequency matches the true signal frequency.

Finally we are able to combine Eqs. 26, 27, and 28 to obtain

$$
\left(\boldsymbol{h}_{1} \mid \boldsymbol{h}_{1}{ }^{\prime}\right) \cong \frac{A}{2} \operatorname{Re}\left[\tilde{W}\left(f-f_{0}\right)\right]
$$

which together with similar calculations for the additional components in Eq. 24 give us

$$
\left(\boldsymbol{h}_{v} \mid \boldsymbol{h}_{\mu}{ }^{\prime}\right) \cong \frac{\mathcal{M}}{2}\left\{\left(\begin{array}{ll}
\mathrm{I} & 0 \\
0 & \mathrm{I}
\end{array}\right) \operatorname{Re}\left[\tilde{W}\left(f-f_{0}\right)\right]+\left(\begin{array}{cc}
0 & \mathrm{I} \\
-\mathrm{I} & 0
\end{array}\right) \operatorname{Im}\left[\tilde{W}\left(f-f_{0}\right)\right]\right\}
$$

as the complete set of inner products between frequencymismatched signal components where I is the $2 \times 2$ identity matrix. Note that when $f=f_{0}$ this expression reduces to Eq. 19.

If we now form the expectation value of the $\mathcal{F}$-statistic (Eq. 19) for mismatched frequencies we find that

$$
\mathrm{E}[2 \mathcal{F}(f)]=4+\rho_{0}^{2}\left|\tilde{W}\left(f-f_{0}\right)\right|^{2} .
$$

Here we see that the fraction of the optimal SNR that contributes to the non-centrality parameter of the $\mathcal{F}$-statistic $\chi^{2}$ distribution is reduced by evaluation of the mod-squared of the antenna-pattern weighted window function with a nonzero argument.

\section{E. Frequency-modulation and the $\mathcal{F}$-statistic}

We now consider the computation of the $\mathcal{F}$-statistic in the case where the data contains a signal from a source in a circular binary orbit but the phase model used in the $\mathcal{F}$-statistic 
template is that of a monochromatic signal of frequency $f$. We again expand $x_{\mu}$ as done in Eq. 24 where no prime indicates the signal and the prime represents the monochromatic template. We again focus on the mismatched signal inner-product $\left(\boldsymbol{h}_{1} \mid \boldsymbol{h}_{1}{ }^{\prime}\right)$ as an example. Starting with Eq. 25 we discard the rapidly oscillating terms inside the integral that will average to zero. We are then left with

$$
\begin{array}{r}
\left(\boldsymbol{h}_{1} \mid \boldsymbol{h}_{1}{ }^{\prime}\right) \cong \frac{1}{2} \sum_{X} \operatorname{Re}\left\{\frac{2}{S_{X}(f)} \int_{0}^{\infty} w_{x}(t) a_{X}^{2}(t) e^{-2 \pi i t\left(f-f_{0}\right)}\right. \\
\left.\exp \left(-2 \pi f_{0} a_{0} \sin \left(\Omega\left(t-t_{a}\right)\right)\right)\right\}
\end{array}
$$

where the final term involving the exponential of a sinusoidal function can be represented using the Jacobi-Anger expansion

$$
e^{i z \sin \theta}=\sum_{n=-\infty}^{\infty} J_{n}(z) e^{i n \theta}
$$

where $J_{n}(z)$ is the $n^{\text {th }}$ order Bessel function of the first kind. This expansion allows us to transform the binary phase term into an infinite sum of harmonics such that we can now write

$$
\begin{aligned}
\left(\boldsymbol{h}_{1} \mid \boldsymbol{h}_{1}{ }^{\prime}\right) & \cong \sum_{n=-\infty}^{\infty} J_{n}\left(2 \pi f_{0} a_{0}\right) \\
& \times \operatorname{Re}\left\{\frac{e^{i \phi_{n}}}{2} \sum_{X} \frac{2}{S_{X}(f)} \int_{0}^{\infty} w_{X}(t) a_{X}^{2}(t) e^{-2 \pi i t\left(f-f_{n}\right)} d t\right\} \\
& \cong \sum_{n=-\infty}^{\infty} J_{n}\left(2 \pi f_{0} a_{0}\right) \frac{A}{2} \operatorname{Re}\left\{\tilde{W}\left(f-f_{n}\right) e^{i \phi_{n}}\right\}
\end{aligned}
$$

It follows that all of the signal components can be expanded in the same way giving us

$$
\begin{gathered}
\left(\boldsymbol{h}_{v} \mid \boldsymbol{h}_{\mu}{ }^{\prime}\right) \cong \frac{\mathcal{M}}{2} \sum_{n=-m_{0}}^{m_{0}} J_{n}\left(2 \pi f_{0} a_{0}\right)\left\{\left(\begin{array}{cc}
\mathrm{I} & 0 \\
0 & \mathrm{I}
\end{array}\right) \operatorname{Re}\left[\tilde{W}\left(f-f_{n}\right) e^{i \phi_{n}}\right]\right. \\
\left.+\left(\begin{array}{cc}
0 & \mathrm{I} \\
-\mathrm{I} & 0
\end{array}\right) \operatorname{Im}\left[\tilde{W}\left(f-f_{n}\right) e^{i \phi_{n}}\right]\right\}
\end{gathered}
$$

where we have truncated the infinite summation (explained below) and defined the monochromatic modulated sideband frequencies and their respective phases as

$$
\begin{aligned}
f_{n} & =f_{0}-n / P_{o}, \\
\phi_{n} & =n \Omega t_{a} .
\end{aligned}
$$

The Jacobi-Anger expansion has allowed us to represent the complex phase of a frequency-modulated signal as an infinite sum of discrete signal harmonics, or sidebands, each separated in frequency by $1 / P_{0} \mathrm{~Hz}$. Each is weighted by the Bessel function of order $n$ where $n$ indexes the harmonics and has a complex phase factor determined by the orbital reference time $t_{a}$. In the limit where the order exceeds the argument, $n \gg z$, the Bessel function rapidly approaches zero allowing approximation of the infinite sum in Eqs. 33 and 34 as a finite sum over the finite range $\left[-m_{0}, m_{0}\right]$ where $m_{0}=\operatorname{cei} 1\left[2 \pi f_{0} a_{0}\right]$. The summation format of Eq. 35 highlights the effects of the binary phase modulation. The signal can be represented as the sum of $M_{0}=2 m_{0}+1$ discrete harmonics at frequencies $f_{n}$ centered on the intrinsic frequency $f_{0}$, where each harmonic peak is separated from the next by $1 / P_{0} \mathrm{~Hz}$.

Combining Eqs. 19, 24, and 35, we can express the expectation value of the $\mathcal{F}$-statistic for a binary signal as a function of search frequency $f$ as

$$
E[2 \mathcal{F}(f)]=4+\rho_{0}^{2} \sum_{n=-m_{0}}^{m_{0}} J_{n}^{2}\left(2 \pi f_{0} a_{0}\right)\left|\tilde{W}\left(f-f_{n}\right)\right|^{2} .
$$

This expression should be interpreted in the following manner. For a given search frequency $f$ the contribution to the non-centrality parameter (the SNR dependent term) is equal to the sum of all sideband contributions at that frequency. Each sideband will contribute a fraction of the total optimal SNR weighted by the $n^{\text {th }}$ order Bessel function squared, but will also be strongly weighted by the window function. The window function will only contribute significantly if the search frequency is close to the sideband frequency. Hence, at a given search frequency close to a sideband, for observation times $\gg P$, the sidebands will be far enough separated in frequency such that only one sideband will contribute to the $\mathcal{F}$-statistic.

\section{F. $C$-statistic}

The $\mathcal{F}$-statistic is numerically maximized over the phase parameters of the signal on a discrete grids. For this search the search frequency $f$ is such a parameter and consequently the $\mathcal{F}$-statistic is computed over a uniformly spaced set of frequency values $f_{j}$ spanning the region of interest. In this section we describe how this $\mathcal{F}$-statistic frequency-series can be used to approximate a search template that is then used to generate a new statistic sensitive to signals from sources in binary systems.

The expectation value of the $\mathcal{F}$-statistic (Eq. 37) resolves into localized spikes at $M_{0}$ frequencies separated by $1 / P_{0} \mathrm{~Hz}$ and centered on the intrinsic gravitational wave frequency $f_{0}$. A template $\mathcal{T}$ based on this pattern with amplitude defined by $G_{n}$, takes the general form

$$
\mathcal{T}(f)=\sum_{n=-m^{\prime}}^{m^{\prime}} G_{n}\left|\tilde{W}\left(f-f_{n}^{\prime}\right)\right|^{2},
$$

with $m^{\prime}=$ ceil $\left[2 \pi f a^{\prime}\right]$ and $f_{n}^{\prime}=f_{0}^{\prime}-n / P^{\prime}$ where we make a distinction between the intrinsic (unknown) values of each parameter (subscript zero) and values selected in the template construction (denoted with a prime). The window function $\tilde{W}$ is dependent only on the times for which data is present and is, therefore, also known exactly.

We define our new detection statistic $C$ as

$$
\begin{aligned}
C(f) & \equiv \sum_{j} 2 \mathcal{F}\left(f_{j}\right) \mathcal{T}\left(f_{j}-f\right) \\
& =(2 \mathcal{F} * \mathcal{T})(f),
\end{aligned}
$$


where the sum over the index $j$ indicates the sum over the discrete frequency bins $f_{j}$ and $f$ is the search frequency. Since the template's "zero frequency" represents the intrinsic gravitational wave frequency, $f$ corresponds to the intrinsic frequency. We see that the $C$-statistic is, in fact, the convolution of the $\mathcal{F}$-statistic with our template, assuming the template is constant with search frequency (an issue we address in the next section).

The benefit of this approach is that the computation of the $\mathcal{F}$-statistic for a known sky position and without accounting for binary effects has relatively low computational cost. Similarly, the construction of a template on the $\mathcal{F}$-statistic is independent of the orbital phase parameter and only weakly dependent upon the orbital semi-major axis and eccentricity. The template is highly dependent upon the orbital period, which, for the sources of interest, is known to high precision. Also, since the $C$-statistic is the result of a convolution, we can make use of the convolution theorem and the speed of the Fast-Fourier-Transform (FFT). Computing $C$ for all frequencies requires only three applications of the FFT. In practice, the $C$-statistic is computed using

$$
\begin{aligned}
& C\left(f_{k}\right)=(2 \mathcal{F} * \mathcal{T})\left(f_{k}\right) \\
& =\sum_{j=0}^{N-1} e^{2 \pi i j k / N}\left(\sum_{p=0}^{N-1} e^{-2 \pi i p j / N} 2 \mathcal{F}\left(f_{p}\right)\right)\left(\sum_{q=0}^{N-1} e^{-2 \pi i j q / N} \mathcal{T}\left(f_{q}\right)\right),
\end{aligned}
$$

which is simply the inverse Fourier transform of the product of the Fourier transforms of the $\mathcal{F}$-statistic and the template. The $\mathcal{F}$-statistic and the template are both sampled on the same uniform frequency grid containing $N$ frequency bins. The $C$ statistic is then also output as a function of the same frequency grid.

\section{G. Choice of $\mathcal{F}$-statistic template}

Treating the $\mathcal{F}$-statistic as the pre-processed input dataset to the $C$-statistic computation, it might be assumed that the optimal choice of template is that which exactly matches the expected form of $2 \mathcal{F}$ in the presence of a signal. As shown in Fig. 2. this approach is highly sensitive to the accuracy with which the projected orbital semi-major axis is known.

We instead propose the use of a far simpler template: one that captures the majority of the information contained within the $\mathcal{F}$-statistic and, by design, is relatively insensitive to the orbital semi-major axis. We explicitly choose

$$
\mathcal{T}_{\mathrm{F}}\left(f_{k}\right)=\sum_{j=-m}^{m} \delta_{k l_{[j]}}
$$

for discrete frequency $f_{k}$, where $\delta_{i j}$ is the Kronecker deltafunction. The frequency index $l$, defined by,

$$
l_{[j]} \equiv \operatorname{round}\left[\frac{j}{P^{\prime} d_{f}}\right] \text {, }
$$

is a function of the best-guess orbital period $P^{\prime}$ and the frequency resolution $d_{f}$. The round[] function returns the integer closest to its argument. The template is therefore composed of the sum of $M=2 m+1$ unit amplitude "spikes" positioned at discrete frequency bins closest to the predicted locations of the frequency-modulated sidebands (relative to the intrinsic gravitational wave frequency). The subscript $F$ refers to the constant amplitude, "Flat" template.

If we now convolve this template with the frequencymodulated $\mathcal{F}$-statistic we obtain the corresponding $C$-statistic, which reduces to

$$
C\left(f_{k}\right)=\sum_{j=-m}^{m} 2 \mathcal{F}\left(f_{k-l_{[j]}}\right) .
$$

Equation 43 is simply the sum of $2 \mathcal{F}$ values taken from discrete frequency bins positioned at the predicted locations of the frequency-modulation sidebands. An example is shown in the right hand panel of Fig. 11, where the most significant $C$ statistic value is located at $f=f_{0}$, and is the point where all sidebands included in the sum contain some signal.

From Eq. 37, the expectation value for the $C$-statistic using the flat-template can be expressed as

$$
\mathrm{E}\left[C\left(f_{k}\right)\right]=4 M+\rho_{0}^{2} \sum_{n=-m}^{m} J_{n}^{2}\left(2 \pi f_{0} a_{0}\right)\left|\tilde{W}\left(f_{n}-f_{k}+l_{[n]} d_{f}\right)\right|^{2},
$$

where the argument of the window function is the frequency difference between the location of the $n^{\text {th }}$ signal sideband and the $n^{\text {th }}$ template sideband on the discrete frequency grid. Note that the $C$-statistic is a sum of $M$ statistically independent non-central $\chi_{4}^{2}$ statistics and hence the result is itself a noncentral $\chi_{4 M}^{2}$ statistic, i.e. with $4 M$ degrees of freedom, where $M=2 \mathrm{ceil}[2 \pi f a]+1$ is the number of sidebands in the expected modulation pattern. The non-centrality parameter is equal to the sum of the non-centrality parameters from each of the summed $2 \mathcal{F}$ values. For a flat template with perfectly matched intrinsic frequency $f=f_{0}$ and orbital period $P^{\prime}=P$, infinite precision $d_{f} \rightarrow 0$, and where the number of sidebands included in the analysis matches or exceeds the true number, the second term in the above equation reduces to $\rho_{0}^{2}$. In this case we will have recovered all of the power from the signal but also significantly increased the contribution from the noise through the incoherent summation of $\mathcal{F}$-statistic from independent frequency bins. In general, where the orbital period is known well, but not exactly, and the frequency resolution is finite, the signal power recovery will be reduced by imperfect sampling of the window function term in Eq. 44, i.e. evaluation at arguments $\neq 0$.

In terms of the generic template defined in equation Eq. 38 . the discrete-frequency flat template is approximately equivalent to the weighting scheme $G_{n}=1$. A more sensitive approach could use

$$
\mathcal{T}_{\mathrm{B}}\left(f_{k}\right)=\sum_{j=-m}^{m} J_{j}^{2}\left(2 \pi f_{0} a_{0}\right) \delta_{k l_{[j]}}
$$

for the template, following the expected form of the $\mathcal{F}$ statistic given in Eq. 37 and using a subscript $\mathrm{B}$ to denote 
Bessel function weighting. Although this would increase sensitivity for closely matched signal templates (constructed with well constrained signal parameters), this performance is highly sensitive to the number of sidebands included in the template and therefore sensitive to the semi-major axis since $M=2$ ceil $[2 \pi f a]+1$. This is mainly due to the "double horned" shape of the expected signal (see the left hand panel of Fig. 11. A large enough offset between the true and assumed semi-major axis will significantly change the template's overlap with the sidebands in the $\mathcal{F}$-statistic and reduce the significance of the $C$-statistic. Considering the semi-major axis is not well constrained for many LMXBs, a search over many templates would be necessary, each with incrementally different semi-major axis values.

The simpler, flat-template (Eq. 41) has the benefit of being far more robust against the semi-major axis uncertainty. In this case the semi-major axis parameter controls only the number of sidebands to use in the template and does not control the weighting applied to each sideband. It also simplifies the statistical properties of the $C$-statistic, making a Bayesian analysis of the output statistics (as described in Section VI) far easier to apply.

The receiver operator characteristic (ROC) curves shown in Fig. 2 compare the performance of the sideband search with both choices of template (flat and Bessel function weighted) for the case of signal with optimal SNR $\rho_{0}=20$. As seen from the figure, the Bessel function weighted template for exact number of sidebands provides improved sensitivity over the flat template. However, when considering the possible (and highly likely) error on the number of sidebands in the template, the performance of the Bessel template is already drastically diminished, even with only a $10 \%$ error on the semimajor axis parameter. It is also interesting to note that for the flat-template the result of an incorrect semi-major axis is asymmetric with respect to an under or over-estimate. The sensitivity degradation is far less pronounced when the template has over-estimated the semi-major axis and, therefore, also over-estimated the number of sidebands. This feature is discussed in more detail in Sec.IVD

\section{H. Approximate binary demodulation}

When a putative source has a highly localized position in the sky, the effect of the Earth's motion with respect to the SSB can be accurately removed from the signal during the calculation of the $\mathcal{F}$-statistic. This leaves only the Doppler modulation from the binary orbit. It is also possible to demodulate the binary orbit (Doppler) modulation in the $\mathcal{F}$-statistic calculation provided the binary orbital parameters $\left(a, P, t_{a}\right)$ are well known. A fully-coherent (sky position- and binary-) demodulated $\mathcal{F}$-statistic search would be very sensitive to any errors in the sky position of binary orbital parameters. It would therefore be necessary to construct a bank of templates spanning the parameter space defined by the uncertainties in these parameters. Adding dimensions to the parameter space increases computational costs and the search becomes unfeasible considering we are already searching over frequency. A

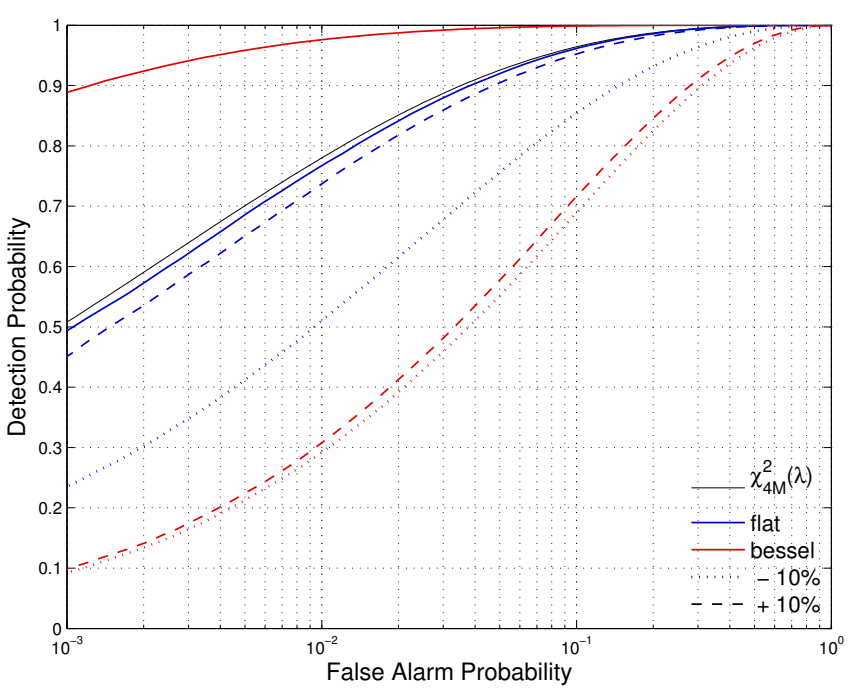

FIG. 2: ROC curves comparing performance of the flat (blue) and Bessel function weighted (red) templates, described by Eqs 41 and 45 respectively. The theoretical (fine black) curve is constructed from a non-central $\chi_{4 M}^{2}(\lambda)$ distributed statistic with non-centrality parameter $\lambda=\rho_{0}^{2}=20$ and represents the expected performance of the flat template. Dashed and dotted curves represent a template with a positive and negative $10 \%$ error on the semi-major axis respectively. Here the signal parameters were chosen such that the number of sidebands were $M=2001$ and curves were constructed using $10^{6}$ realizations of noise.

fully coherent search of this type would be possible for known sources with known emission frequencies, for example pulsing sources like millisecond pulsars (MSPs).

In this section we show how prior information regarding the binary orbit of a source can be used to increase the sensitivity of our semi-coherent approach, without increasing computational costs. By performing a "best guess" binary phase demodulation within the $\mathcal{F}$-statistic, we show that the number of sidebands in the template is reduced by a factor proportional to the fractional uncertainty in the orbital semi-major axis. Consequently a reduction in the number of sidebands increases the sensitivity of the search by reducing the number of degrees of freedom (see Sec. VI).

Expressing our current best estimate for each parameter $\boldsymbol{\theta}$ as the sum of the true value $\boldsymbol{\theta}_{0}$ and an error $\Delta \boldsymbol{\theta}_{0}$, such that

$$
\boldsymbol{\theta}=\boldsymbol{\theta}_{0}+\Delta \boldsymbol{\theta}_{0}
$$

we can determine the phase offset of the binary orbit from the error in the binary orbital parameters. The offset in phase is the difference between the true and best estimate binary phase and using Eq. $12 \mathrm{~b}$ can be approximated by

$$
\begin{aligned}
\Delta \phi_{\text {bin }} & \simeq-2 \pi f\left\{\left(a_{0} \Delta f+f \Delta a_{0}\right) \sin \left[\Omega_{0}\left(t-t_{a}\right)\right]\right. \\
& \left.+\left[f a_{0}\left(\Delta \Omega_{0}\left(t-t_{\mathrm{a}}\right)-\Omega_{0} \Delta t_{a}\right)\right] \cos \left[\Omega_{0}\left(t-t_{a}\right)\right]\right\}+O\left(\Delta \boldsymbol{\theta}^{2}\right) \\
& \simeq-2 \pi f \Delta a_{0} \kappa \sin \left[\Omega_{0}\left(t-t_{a}\right)+\gamma\right],
\end{aligned}
$$


with

$$
\begin{aligned}
& \kappa=\sqrt{\left(1+\frac{a_{0}}{\Delta a_{0}} \frac{\Delta f}{f}\right)^{2}+\left(\frac{a_{0}}{\Delta a_{0}}\left(\Delta \Omega_{0}\left(t-t_{a}\right)-\Omega_{0} \Delta t_{a}\right)\right)^{2}}, \\
& \gamma=\tan ^{-1}\left[\frac{\Delta \Omega_{0}\left(t-t_{a}\right)-\Omega_{0} \Delta t_{\mathrm{a}}}{\left(\Delta a_{0} / a_{0}\right)-(\Delta f / f)}\right]+ \begin{cases}0 & \text { if }\left(\frac{\Delta a_{0}}{a_{0}}\right)-\left(\frac{\Delta f}{f}\right) \geq 0 \\
\pi & \text { if }\left(\frac{\Delta a_{0}}{a_{0}}\right)-\left(\frac{\Delta f}{f}\right)<0 .\end{cases}
\end{aligned}
$$

Here we have expanded the binary phase difference to leading order in the parameter uncertainties and obtained a phase expression similar in form to the original binary phase. In the specific regime where the fractional uncertainty in the orbital semi-major axis far exceeds the fractional uncertainty in the intrinsic frequency we see that the first term in Eq. 48 becomes $\approx 1$. Similarly, if the fractional uncertainty in the orbital semi-major axis also far exceeds the deviation in orbital angular position $\Delta \Omega\left(t-t_{a}\right)-\Omega \Delta t_{a}$ then the second term $\approx 0$. This is generally the case for the known LMXBs (see Table I) and in this regime $\kappa$ can be accurately approximated as unity, yielding

$$
\Delta \phi_{\text {bin }} \approx-2 \pi f \Delta a_{0} \sin \left[\Omega\left(t-t_{a}\right)+\gamma\right] .
$$

Hence, after approximate binary demodulation, the argument of the Bessel function and the summation limits in the expected form of the $\mathcal{F}$-statistic (in Eq. 37 for example) can be replaced with $\Delta z_{0}=2 \pi f \Delta a_{0}$. The number of frequencymodulated sidebands is now reduced by a factor of $\Delta a_{0} / a_{0}<$ 1. We must stress that $\Delta a$ is an unknown quantity and is the difference between the best estimate value of $a$ and the true value $a_{0}$. The $\mathcal{F}$-statistic after such a demodulation process will therefore have a reduced but unknown number of sidebands, although it will still retain the standard sideband frequency spacing $1 / P$. The sideband phasing $\phi_{n}$ will also be unknown due to the presence of the phase term $\gamma$ but is of no consequence to the search since the $\mathcal{F}$-statistic is insensitive to phase.

\section{PARAMETER SPACE}

In this section we will discuss each of the parameters involved in the search and how the search sensitivity depends upon the uncertainty in these parameters. Demodulation of the signal phase due to the Earth's motion requires accurate knowledge of the source sky position. If the observation time is long enough, we need to consider the sky position as a search parameter, as discussed in Sec. IV A The gravitational wave frequency is the primary search parameter. In Sec.IVB we discuss the limitations on our search strategy due to its uncertainty. The orbital period and semi-major axis are discussed in Sections IV C and IV D respectively. The effects of ignoring the orbital eccentricity are discussed in Sec. IVE

\section{A. Sky position and proper motion}

In order to quantify the allowable uncertainty in sky position we will define a simplistic model describing the phase
$\Psi(t)$ received at Earth from a monochromatic source at infinity at sky position $(\alpha, \delta)$. If we neglect the detector motion due to the spin of the Earth and consider only the Earth's orbital motion then we have

$$
\Psi(t)=2 \pi f_{0}\left[t+R_{\oplus} \cos \delta \cos \left(\Omega_{\oplus}\left(t-t_{\mathrm{ref}}\right)+\alpha\right)\right],
$$

where $f_{0}$ is the signal frequency, $\alpha$ and $\delta$ are the true right ascension and declination and $R_{\oplus}$ and $\Omega_{\oplus}$ are the distance of the Earth from the SSB and the Earth's orbital angular frequency respectively. We also define a reference time $t_{\text {ref }}$ that represents the time at which the detector passes through the vernal equinox. For an observed sky position $\left(\alpha^{\prime}, \delta^{\prime}\right)=$ $(\alpha+\Delta \alpha, \delta+\Delta \delta)$ the corresponding phase offset $\Delta \Psi(t, \Delta \alpha, \Delta \delta)=$ $\Psi\left(t, \alpha^{\prime}, \delta^{\prime}\right)-\Psi(t, \alpha, \delta)$ amounts to

$$
\begin{aligned}
\Delta \Psi \approx & -2 \pi f_{0} R_{\oplus}\left[\Delta \delta \sin \delta \cos \left(\Omega_{\oplus}\left(t-t_{\mathrm{ref}}\right)+\alpha\right)\right. \\
& \left.+\Delta \alpha \cos \delta \sin \left(\Omega_{\oplus}\left(t-t_{\mathrm{ref}}\right)+\alpha\right)\right],
\end{aligned}
$$

where we have expanded the expression to leading order in the sky position errors. We now make the reasonable assumption that our analysis would be unable to tolerate a deviation in phase between the signal and our template of more than $O(1)$ radian over the course of an observation on the same timescale of the Earth's orbit.

If we also notice that the worst case scenario (smallest allowable sky position errors) corresponds to sky positions for which the trigonometric terms in the previous expression are largest, i.e. of order unity, then we have

$$
|\Delta \alpha, \Delta \delta| \leq\left(2 \pi f_{0} R_{\oplus}\right)^{-1}
$$

If we consider signals of frequency $1 \mathrm{kHz}$, this gives a maximum allowable sky position offset of $|\Delta \alpha, \Delta \delta| \simeq 100$ mas. This expression also validates our model assumption that the sky position sensitivity to the Earth spin would be dominated by the effect from the Earth orbit for long observation times.

A similar argument can be made for the proper motion of the source where we would be safe to model the sky position as fixed if the change $(\Delta \alpha, \Delta \delta)=\left(\mu_{\alpha}, \mu_{\delta}\right) T_{\mathrm{s}}$, over the course of the observation also satisfied Eq. 53 .

\section{B. Spin frequency}

The spin frequency $v_{\mathrm{s}}$ of some LMXBs can be directly measured from X-ray pulsations, believed to originate from a hotspot on the stellar surface, where accreted material is funneled onto the magnetic pole with the magnetic axis generally misaligned with the spin axis. X-ray pulsations have been observed in 13 LMXB systems so far, three of which are intermittent [66].

Some LMXBs exhibit recurrent thermonuclear X-ray bursts. Fourier spectra reveal oscillations during the rise and tail of many bursts, which are believed to originate from asymmetric brightness patterns on the stellar surface. In seven LMXBs which exhibit both pulsations and bursts, the asymptotic burst oscillation frequency at late times matches the pulse 
frequency. Where there are no pulsations, many bursts need to be observed to measure the asymptotic burst oscillation reliably. The spin frequency of an additional ten systems has been determined from burst oscillations only [67], but due to the uncertainties involved, are usually quoted to within uncertainties of $\pm(5-10) \mathrm{Hz}$.

Another class of LMXBs exhibit high frequency quasiperiodic oscillationss (QPOs) in their persistent X-ray emission. These $\mathrm{kHz}$ QPOs usually come in pairs, although singles and triples are occasionally observed and the QPO peak frequencies usually change over time. In some cases the separation of the QPO peaks is roughly constant, but this is not always the case [68-70]. For the few QPO systems where $v_{\mathrm{S}}$ can be determined from pulses or burst oscillations there has been no evidence suggesting consistency with an existing QPO model that links the QPO and spin frequencies. For our purposes, $v_{\mathrm{s}}$ is considered unknown in sources without pulsations or confirmed bursts.

In addition to potentially broad uncertainties in $v_{\mathrm{s}}$, we know little about how its value may fluctuate over time due to accretion. Changes in the accretion flow will exert a time varying torque on the star which will result in a stochastic wandering of the spin frequency. In this case the signal can no longer be assumed monochromatic over a given observation time. To quantify the resulting phase wandering, we assume that the fluctuating component of the torque $\delta N_{\mathrm{a}}$ flips sign randomly on the timescale $\tau_{\mathrm{s}}$ consistent with the inferred variation in accretion rate. If the mean torque $N_{\mathrm{a}}=\dot{M}(G M R)^{1 / 2}$ due to steady-state disk-fed accretion, then the angular spin frequency $\Omega_{s}=2 \pi v_{\mathrm{s}}$ experiences a random walk with step size $\left(\delta N_{\mathrm{a}} / I\right) \tau_{\mathrm{s}}$, where $I$ is the stellar moment of inertia. After time $T_{\mathrm{s}}$, the root-mean-square drift is

$$
\left\langle(\delta \Omega)^{2}\right\rangle^{1 / 2}=\left(T_{\mathrm{s}} / \tau_{\mathrm{s}}\right)^{1 / 2} \frac{\delta N_{\mathrm{a}} \tau_{\mathrm{s}}}{I} .
$$

This frequency drift will wander outside a Fourier frequency bin width if $\left\langle(\delta \Omega)^{2}\right\rangle^{1 / 2}>2 \pi / T_{\mathrm{s}}$. If we choose $\tau_{\mathrm{s}}$ such that the accretion rate can vary up to a factor of two in this time, then the worst case $\delta N_{\mathrm{a}}=N_{\mathrm{a}}$ leads to the restriction

$$
T_{\mathrm{s}}^{\mathrm{spin}}<\frac{(2 \pi)^{2 / 3}}{(G M R)^{1 / 3}}\left(\frac{I}{\dot{M}}\right)^{2 / 3}\left(\frac{1}{\tau_{\mathrm{s}}}\right)^{1 / 3} .
$$

This is the primary reason why an application of the the basic sideband search, as described here, must be limited in the length of data it is allowed to analyse. By exceeding this limit it becomes increasingly likely that the spin wandering inherent to a true signal will cause signal power to leak between adjacent frequency bins. Consequently the assumption that $\mathcal{F}$-statistic signal power is localized in frequency-modulated sidebands will become invalid and the sensitivity of the $C$ statistic will deteriorate.

\section{Orbital Period}

The sideband search relies on relatively precise electromagnetic (EM) measurements of the orbital period in order to construct a search template. The duration of the orbit defines the minimum observation time, since $T \gtrsim 3 P / 2$ is required before sidebands become clearly resolved in the spectrum [60]. The uncertainty in the orbital period will determine the number of templates required to fully sample the search space, or equivalently, the maximum observation time allowed for a single value of $P$.

We will now provide an indication of the sensitivity of the search to errors in the orbital period. If our estimate (observation) of the orbital period $P$ is offset from the true value $P_{0}$ by an amount $\Delta P$, we would expect the error to seriously affect the $C$-statistic recovered from the search once it is large enough to shift the outermost "tooth" in the sideband template by one canonical frequency bin away from the true sideband location. In this case, the offset between the template and true sideband frequency is proportional to the number of sidebands from the central spike. There will be low mismatch at the center of the template extending to $O(100 \%)$ mismatch at the edges. It follows that the average signal power recovered from such a mismatched template will be $O(50 \%)$ and therefore serves as a useful threshold by which to determine the maximum allowed $\Delta P$.

If we use the measured value of $P$ as our template parameter, the template centered at frequency $f$ then consists of $\simeq 4 \pi f a$ unit spikes (or teeth) separated by $1 / P$. Assuming that the central spike is exactly equal to the true intrinsic gravitational wave frequency, any errors in the orbital period will be propagated along the comb, causing the offset between the true and template frequency of any particular sideband to grow progressively larger. The frequency difference $\Delta f_{P}$ between the outermost template sideband, at frequency $f+2 \pi f a / P^{\prime}$, and the outermost signal sideband at $f+2 \pi f a / P$, is given by

$$
\Delta f_{P} \approx 2 \pi f a\left(\frac{|\Delta P|}{P^{2}}\right),
$$

for $\Delta P \ll P$. To satisfy the condition described above we now require that this frequency shift should be less than the size of one frequency bin. The true frequency bin size $d_{f}$ is determined by the observation time span and is given by

$$
d_{f}=\frac{1}{r T_{\mathrm{s}}}
$$

where $r$ is the resolution used in the $\mathcal{F}$-statistic calculation. ${ }^{2}$ Using Eqs. 56 and 57 and imposing the condition that $\Delta f_{P}<$ $d_{f}$ provides an estimate for the maximum allowable (orbital period limited) coherent observation timespan,

$$
T_{\mathrm{s}}^{P} \approx \frac{P^{2}}{2 \pi f a|\Delta P|} .
$$

Given a relatively poorly constrained orbital period uncertainty, this restriction may provide too short a duration. This could be because it is then in conflict with the requirement that $T>3 P / 2$ or simply because more SNR is desired from

\footnotetext{
${ }^{2}$ The default resolution factor is $r=2$
} 
the signal. In either case, the orbital period space must then be divided into templates with spacing $\delta P$ derived from simply rearranging Eq. 58 to solve for $\Delta P$. In relative terms the sideband search places very strong constraints on the prior knowledge of the orbital period compared to the other search parameters.

\section{Semi-major axis}

An error in the value of the orbital semi-major axis results in an incorrect choice for the number of sidebands in the template. As can be seen in Eq. 44, an underestimate results in the summation of a fraction of the total power in the signal whereas an overestimate results in a dilution of the total power by summing additional noise from sideband frequencies containing no signal contribution.

If we define the true semi-major axis parameter $a_{0}$ as the measured value $a$ and some (unknown) fraction $\xi$ (where $\xi \in \mathbb{R}$ ) of the measurement error $\Delta a$ (i.e. $a_{0}=a+\xi \Delta a$ ), we can investigate the effects of errors on the semi-major axis parameter in terms of this offset parameter $\xi$. We consider the advantage of using a deliberately offset value $a^{\prime}$ instead of the observed value $a$ in order to minimize losses in recovered SNR.

The ROC curves shown in Fig. 3 show the effects of these offsets, and clearly illustrate degradation in the performance of the $C$-statistic as $|\Delta a|(|\xi|)$ increases. The reduction in detection confidence at a given false alarm probability is much faster for $a^{\prime}<a_{0}(\xi<0)$, when the template underestimates the width of the sideband structure, than for $a^{\prime}>a_{0}(\xi>0)$. This is natural considering the "horned" shape of the signal (see the left hand panel of Fig. 1 and Sec. IIIG). Although it is already clear from this figure that the performance of the search is not symmetric about $a^{\prime}=a_{0}$, this asymmetry is much better illustrated in Fig. 4 where for different values of the false alarm rate we show the detection probability plotted against the offset parameter $\xi$.

This plot provides us with a rough scheme by which to improve the search performance by exploiting the asymmetry in search sensitivity with respect to $\xi$. In general, we are keen to probe the low false alarm and high detection probability regime in which it is clear that using a template based on an orbital semi-major axis value $>$ the best estimate reduces the possibility that the bulk of the signal power (in the horns) will be missed. Based on Fig. 4 we choose

$$
a^{\prime}=a+\Delta a
$$

as our choice of semi-major axis with which to generate the search template.

\section{E. Orbital eccentricity}

The orbital eccentricity $e$ of the LMXB sources is expected to be highly circularized $\left(e<10^{-3}\right)$ by the time mass transfer occurs within the system. In Eq. 10 we give the first-order

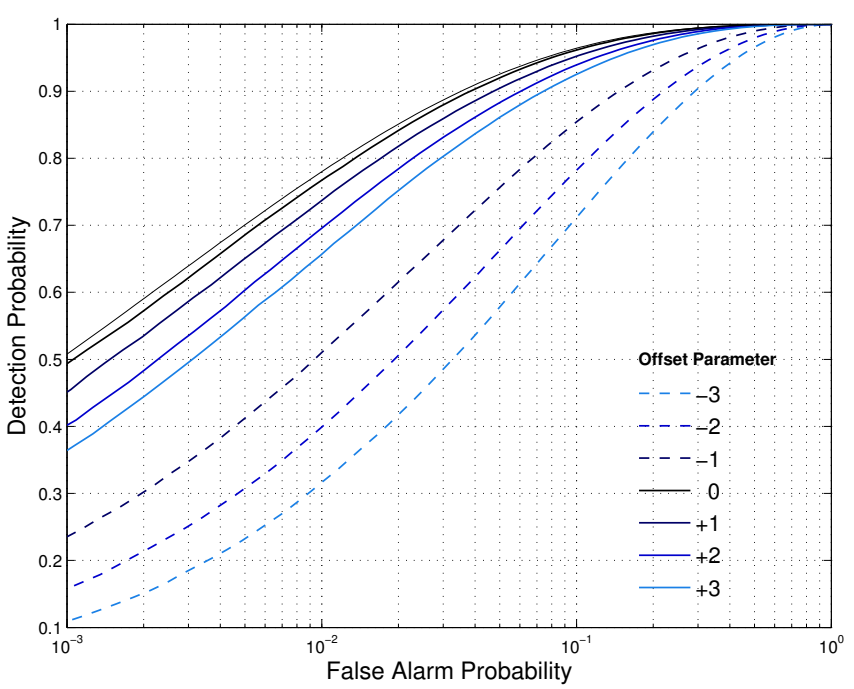

FIG. 3: ROC curves showing how the performance of the flat template $C$-statistic is affected by an offset in the orbital semi-major axis assuming it is measured exactly (i.e. $a=a_{0}$ ). The thick black curve represents a zero offset $(\xi=0)$. Thick colored curves represent a positive offset in the semi-major axis $(\xi>0)$. Dashed colored curves represent negative offsets $(\xi<0)$. The fainter black curve is constructed from a statistic governed by a non-central $\chi_{4 M}^{2}(\lambda)$ distribution with a non-centrality parameter $\lambda=\rho_{0}^{2}$ and represents the theoretical expected behavior of a perfectly matched template. Signal parameters are the same as described in Fig. 2. with $\rho_{0}=20$, $M=2001$ and using $10^{6}$ realizations of noise.

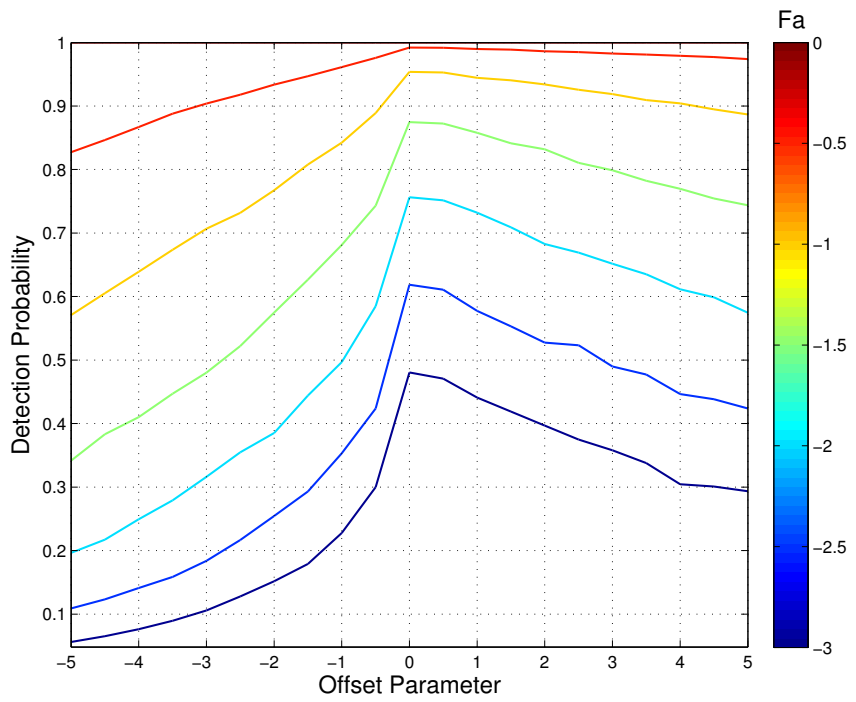

FIG. 4: Performance of the $C$-statistic with respect to offsets in the semi-major axis at given false alarm probability $F_{a}$. The offset parameter $\xi$ quantifies the semi-major axis error in terms of known parameters $a$ and $\Delta a$. The color-bar represents the log of the false alarm probability, ranging from $10^{-3}$ (bottom, blue) to 0 (top, red). Signal parameters are the same as Figs. 2 and 3, with $\rho_{0}=20, M=2001$ and a $\Delta a / a=10 \%$ fractional uncertainty on the semi-major axis, using $10^{6}$ realizations of noise. 
correction (proportional to $e$ ) of the retarded time at the SSB. If we include higher order terms in the expansion, the phase (Eq.11) can be written as

$$
\begin{array}{r}
\Phi(t) \simeq 2 \pi f_{0} a_{0}\left\{\sum_{k=1}^{\infty} c_{k} \sin \omega \cos \left[k \Omega\left(t-t_{p}\right)\right]\right. \\
\left.+d_{k} \cos \omega \sin \left[k \Omega\left(t-t_{p}\right)\right]\right\},
\end{array}
$$

where the first 4 coefficients (expanded to $O\left(e^{4}\right)$ ) in the sum are given by

$$
\begin{aligned}
& c_{1}=1-\frac{3}{8} e^{2}+\frac{5}{192} e^{4}+O\left(e^{6}\right) \\
& d_{1}=1-\frac{5}{8} e^{2}-\frac{11}{192} e^{4}+O\left(e^{6}\right) \\
& c_{2}=\frac{1}{2} e-\frac{1}{3} e^{3}+O\left(e^{5}\right) \\
& d_{2}=\frac{1}{2} e-\frac{5}{12} e^{3}+O\left(e^{5}\right) .
\end{aligned}
$$

Hence the phase for $e \neq 0$ is a sum of harmonics of the orbital frequency. When including additional eccentric phase components in this way, the sum inside the exponential can be expressed as a product of sums such that the Jacobi-Anger expansion (Eq. 33) can be modified such that

$$
\exp \left[i \sum_{k} z_{k} \sin k \theta\right]=\prod_{k=1}^{\infty} \sum_{n=-\infty}^{\infty} J_{n}\left(z_{k}\right) e^{i n k \theta}
$$

where $z_{k}$ corresponds to the $k^{\text {th }}$ amplitude term (on the left hand side) that defines the argument of the Bessel function for each $k$ in the product (on the right hand side). Equation 62 tells us that eccentric signals can be thought of in a similar way to circular orbit cases. The signal can be modeled as being composed of many harmonics all separated by some integer number of the inverse of the orbital period. In the eccentric case $k$ is allowed to be $>1$ and power can be spread over a far greater range of harmonics. What is important to note however, is that the signal power remains restricted to those discrete harmonics.

If we consider only leading order terms in the eccentricity expansion (as in Eq. 10) the form of the Jacobi-Anger expression given above becomes the product of 2 sums where we consider only $k=1,2$. The $k=1$ terms are simply the circular orbit terms and describe how the signal power is distributed amongst $\approx 2 z_{1}$ sidebands at frequencies offset from the intrinsic source frequency by integer multiples of $\theta$.

In our low eccentricity case we notice that the next to leading order term in the expansion, $k=2$, has a corresponding Bessel function argument of $O\left(z_{1} e\right)$ and will therefore have far fewer, $\sim 2 z_{1} e$, non-negligible terms in sum over $n$. Taking the product between the $k=1$ and $k=2$ sums will then produce a redistribution of the signal power amongst a slightly expanded range of harmonic frequencies. For the circular orbit case we expect power to be spread amongst $\approx 2 z_{1}$ sidebands whereas we now expect the same power to be divided amongst $\approx 2 z_{1}(1+2 e)$ sidebands.
In general, orbital eccentricity causes a redistribution of signal power amongst the existing circular orbit sidebands and will cause negligible leakage of signal power into additional sidebands at the boundaries of the sideband structure. Orbital eccentricity also has the effect of modifying the phase of each sideband. However, as shown in Section IIIE, the standard sideband search is insensitive to the phase of individual sidebands.

\section{PRIMARY SOURCES}

The benefit of the sideband search is that it is robust and computationally cheap enough to be run over a wide frequency band [59]. The most suitable targets are those with well-measured sky position and orbital periods, reasonably well constrained semi-major axes, and poorly or unconstrained spin frequency.

The most suitable candidates in terms of these criteria are LMXBs due to their high accretion rate (directly related to gravitational wave amplitude) and their visibility in the electromagnetic regime (predominantly X-ray, but optical and radio observations also provide accurate sky position, ephemeris and sometimes orbital information). They are classified into three main types depending on the behavior of their X-ray emissions: pulsing, bursting or QPO sources. Pulsating and frequently bursting LMXBs usually have a well determined spin frequency and are better suited to the more sensitive, narrow-band techniques, such as LIGO's known pulsar pipeline [43, 44] including corrections for the binary motion. Non-pulsing burst sources with irregular or infrequent bursts still have a fairly wide $(\mathrm{O}$ a few $\mathrm{Hz})$ range around the suspected spin frequency. A convincing relationship between QPOs and the spin frequency of the neutron star has not yet been determined, so the spin frequency of purely QPO sources is considered unknown for our applications.

The gravitational wave strain amplitude is directly proportional to the square root of the $\mathrm{x}$-ray flux $h_{0} \propto F_{\mathrm{x}}^{1 / 2}$ (Eq. 7), so the most luminous sources, which are usually the quasi-periodic oscillation (QPO) sources, will be the most detectable. In addition, the (already weak) gravitational wave strain amplitude is proportional to the inverse of the distance to the source, so closer (i.e. galactic) sources are also favorable.

In this section we present possible sources to which the sideband search can be applied. We start with galactic LMXBs and consider the most detectable sources in terms of their parameter constraints. We exclusively consider sources requiring wide frequency search bands $(\gtrsim 5 \mathrm{~Hz}$ ), and so neglect the accreting millisecond pulsars. The detectability of a wider range of accreting sources, with some measurement or estimate of spin frequency, in terms of general gravitational wave searches was reviewed in [28]. 
TABLE I: Target sources for the sideband method. The different columns list the X-ray flux $F_{X}$ (in units of $10^{-8} \mathrm{erg} \mathrm{cm}^{-2} \mathrm{~s}^{-1}$ ), distance $d$, sky position uncertainty $\Delta \beta$, fractional error on the semi-major axis $\Delta a / a$ and orbital period $\Delta P / P$, and the orbital period limited observation time at a frequency of $\left.1 \mathrm{kHz} T_{\mathrm{s}}^{P}\right|_{1 \mathrm{kHz}}$. The horizontal line separates QPO (top) and burst (bottom) sources.

\begin{tabular}{lcccccc}
\hline \hline Source & $F_{\mathrm{X}}\left(F_{*}\right)^{a}$ & $\mathrm{~d}(\mathrm{kpc})$ & $\Delta \beta(\operatorname{arc~sec})$ & $\Delta a / a$ & $(\Delta P / P)\left(\times 10^{-7}\right)$ & $\left.T_{\mathrm{s}}^{P}\right|_{1 \mathrm{kHz}}$ \\
\hline Sco X-1 & 40 & 2.8 & $3 \times 10^{-4}$ & 0.13 & 50 days \\
4U 1820-30 & 2.1 & 7.4 & 0.15 & 0.48 & 2 & 300 days \\
Cyg X-2 & 1.1 & 10.55 & 0.5 & 0.12 & 0.004 & 0.9 \\
J 2123-058 & 0.21 & 9.6 & 0.6 & 0.19 & 10 & 3.5 years \\
\hline 4U 1636-536 & 0.84 & 6 & $<60$ & 0.82 & 0.1 & 500 \\
X 1658-298 & 0.67 & 12 & 0.1 & 0.12 & 6 & 5 years \\
XB 1254-690 & 0.09 & 13 & 0.6 & 0.77 & 3 hours \\
EXO 0748-676 & 0.036 & 7.4 & 0.7 & 0.72 & 40 days \\
4U 1916-053 & 0.027 & 8 & 0.6 & & \\
\hline \hline
\end{tabular}

${ }^{a} F_{*}=10^{-8} \mathrm{erg} \mathrm{cm}^{-2} \mathrm{~s}^{-1}$

\section{A. Galactic LMXBs}

The sideband search is best suited to LMXBs with a relatively large uncertainty in the spin frequency ( $~ a$ few $\mathrm{Hz}$ ), so QPO and poorly constrained burst sources are the best targets. The requirement of a relatively well defined sky position and orbital period excludes many sources including those that are considered to be X-ray bright. Table I lists some of the galactic LMXBs, and their limiting parameters, for which the sideband search is most applicable. The parameters displayed in the table allow us to determine the most suitable targets for the search.

For each source the table lists the bolometric X-ray flux $F_{\mathrm{X}}$, the distance to the source $d$, the error in the sky position $\Delta \beta=(\Delta \alpha, \Delta \delta)$, the fractional error in the semi-major axis $\Delta a / a$ and the orbital period limited observation time $\left.T_{\mathrm{s}}^{P}\right|_{1 \mathrm{kHz}}$ calculated using Eq. 58 at a frequency of $1 \mathrm{kHz}$. Although we could expect gravitational wave emission up to $\sim 1500 \mathrm{~Hz}$ (from the currently measured spin distributions of LMXBs maxing out at $\sim 720 \mathrm{~Hz}$ ), $1 \mathrm{kHz}$ is chosen as an upper bound on the search frequency since the sensitivity of LIGO detectors is limited at high frequencies and the amplitudes of these systems are not expected to be very strong. Sources with poorly constrained $(\Delta a / a>0.9)$ semi-major axis and sky position $\left(\Delta \beta>60^{\prime \prime}\right)$ have not been included. The sources are listed in order of their bolometric X-ray flux within each source group with QPO sources in the top and burst sources in the bottom half of the table. The distance is included as a reference but is already taken into account in calculation of $F_{\mathrm{X}}$. From these factors alone, Sco X-1 is already the leading candidate source. The sky position error $\Delta \beta$ should be less than 100 mas for a source with $f_{0}=1 \mathrm{kHz}$ (see Sec. IV A). Sco X-1 is the only candidate that falls easily within this basic limit, although a few other sources are borderline cases. The fractional error in semi-major axis is included also as a guide. Although a smaller error on this parameter improves our sensitivity, as shown in Sec. IV D we are relatively insensitive to $a$ uncertainties on the scale of 10's of percent. The final column lists the orbital period limited observation timespan $T_{\mathrm{s}}^{P}$ at a frequency of $1 \mathrm{kHz}$. Although the spin frequencies of the burst sources are better constrained than QPO sources, the compar- ison of $T_{\mathrm{s}}^{P}$ is still made at $1 \mathrm{kHz}$ so that a direct comparison on the source parameters (rather than search performance) can be made. This column is included for reference as the orbital period may not be the tightest constraint on the observation time (c.f. Sec. IV B. It does, however, give an indication of how well the orbital period of the source is constrained and specifically how it affects the search performance.

\section{B. Sco X-1}

Sco X-1, the first LMXB to be discovered, is also the brightest extra-solar X-ray source in the sky. The direct relation between gravitational wave strain and x-ray flux given by Eq. 7 makes it also the most likely to be a strong gravitational wave emitter. This, as well as the parameter constraints displayed in Table I, makes it an ideal candidate for the sideband search. Table II provides a list of Sco X-1 parameters determined from various electromagnetic observations. The table includes the parameters required to run the sideband search together with some values used for calculating limits and constraints on the performance and sensitivity of the search. The bottom section of the table lists some of the limits and constraints derived using the above mentioned parameters.

Running the standard version of the sideband search requires accurate knowledge of the sky position and orbital period and approximate knowledge of the semi-major axis. The sky position $\beta=(\alpha, \delta)$ listed for Sco X-1 is accurate to within 0.3 mas. This error is well within the 100 mas limit defined in Sec. IV A justifying the assumption of a fixed sky position. The accuracy of measurements of the orbital period require only a single sideband template if the observation timespan is within $T_{\mathrm{s}}<T_{\mathrm{s}}^{\mathrm{obs}} \approx 49$ days (for Sco X-1 at $1 \mathrm{kHz}$ ). The semi-major axis and its measurement error are also required for construction of the sideband template.

Estimates of the primary (accreting neutron star) and secondary (donor star) masses, as well measurements of the bolometric X-ray flux $\left(F_{\mathrm{X}}\right)$ are required to estimate the indirect, torque balance, gravitational wave strain upper limit $h_{0}^{\mathrm{EQ}}$ using Eq. 7. displayed in the bottom section of the table. The spin frequency limited observation timespan is also listed here and 
TABLE II: Sco X-1 system parameters required for the sideband search. Directly observable parameters are presented in the top half of the table. The bottom half, separated by the horizontal line, displays search limits and constraints derived from these.

\begin{tabular}{|c|c|c|c|c|c|}
\hline Parameter & Symbol & Value & Units & Uncertainty & References \\
\hline Right Ascension & $\alpha$ & $16^{h} 19^{m} 55^{s} .0850$ & mas & \pm 0.3 & {$[71]$} \\
\hline Declination & $\delta$ & $-15^{\circ} 38^{\prime} 24.9^{\prime \prime}$ & mas & \pm 0.3 & 71 \\
\hline Proper motion & $\mu$ & 14.1 & mas $\mathrm{yr}^{-1}$ & & 71 \\
\hline Parallax & $\pi_{\beta}$ & 0.36 & mas & \pm 0.04 & 71 \\
\hline Moment of inertia & $I$ & $10^{38}$ & $\mathrm{~kg} \mathrm{~m}^{2}$ & & [71] \\
\hline Accretion rate & $\dot{M}$ & $1.23 \times 10^{15}$ & $\mathrm{~kg} \mathrm{~s}^{-1}$ & & [71 \\
\hline Bolometric X-ray flux & $F_{\mathrm{X}}$ & $40 \times 10^{-8}$ & 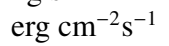 & & 28 \\
\hline Projected semi-major axis light travel time & $a$ & 1.44 & $\mathrm{~s}$ & \pm 0.18 & 72 \\
\hline Orbital Period & $P$ & 68023.82 & $\mathrm{~s}$ & \pm 0.06048 & 73 \\
\hline NS spin inclination angle & $\iota$ & 44 & $\operatorname{deg}$ & \pm 6 & 74] \\
\hline GW polarization angle & $\psi$ & 234 & deg & \pm 3 & 74 \\
\hline Time of periapse passage (SSB) & $t_{p}$ & 614638484 & $\mathrm{~s}$ & \pm 400 & 72, 75 \\
\hline Strain amplitude (at $v_{\mathrm{s}}=300 \mathrm{~Hz}$ ) & $h_{0}^{300}$ & $3.5 \times 10^{-26}$ & & & Eq. 7 \\
\hline Spin limited observation timespan & $T_{\mathrm{s}}^{\mathrm{spin}}$ & 13 & days & & Eq. 55 \\
\hline
\end{tabular}

requires values for the accretion rate $\dot{M}$ and moment of inertia $I$ to calculate this value for Sco X-1 using Eq. 55 assuming a spin-wandering timescale $\tau_{\mathrm{s}}=1$ day. ${ }^{3}$ The corresponding value of $T_{\mathrm{s}}^{\text {spin }} \approx 13$ days displayed in the table is more restrictive than the orbital period limited timespan and is our limiting time constraint in the search.

\section{STATISTICAL ANALYSIS}

Let us first assume that our analysis has yielded no significant candidate signal given a designated significance threshold. In this case, with no evidence for detection, we place an upper limit on the possible strength of an underlying signal. In the literature on continuous-wave gravitational signals, it is common to determine these upper limits numerically [53, 78, 79] or semi-analytically [46, 80] using frequentist Monte Carlo methods. In these cases simulated signals are repeatedly added to data over a range of frequencies and recovered using a localized, computationally cheap, search around the point of injection.

The sideband algorithm combines signal from many (typically $\sim 10^{3}$ ) correlated $\mathcal{F}$-statistic frequency bins which must be computed over a relatively wide frequency band for each simulated signal. Such computations represent a computational cost far in excess of existing methods and are only manageable for a small parameter space, e.g. injection studies where the signal frequency is known and $O\left(10^{2}\right)$ realizations are feasible. The computations become daunting for a wideband search covering more than a few $\mathrm{Hz}$.

We choose to optimize the process by calculating upper

\footnotetext{
${ }^{3}$ Assuming the instantaneous accretion rate does not vary more than the $\mathrm{x}$-ray flux, observations of the $\mathrm{x}$-ray variability of Sco X-1 show that the accretion rate can vary by roughly a factor of two over a timescale $\tau_{\mathrm{s}}=1$ day. [76 77]
}

limits within a Bayesian framework. This is an especially appealing alternative since the probability density function (PDF) of the $C$-statistic takes a relatively simple, closed, analytic form. Bayesian upper limits have been computed in timedomain gravitational wave searches targeting known sources (pulsars) [43, 45, 81], and cross-correlation searches for the stochastic background [55, 56, 82]. Comparisons on specific data sets have shown that Bayesian and frequentist upper limits are consistent [42, 45, 83].

\section{A. Bayes Theorem}

In the Bayesian framework, the posterior probability density of the hypothesis $H$ given the data $D$ and our background information $I$ is defined as

$$
p(\boldsymbol{\theta} \mid D, H, I)=p(\boldsymbol{\theta} \mid H, I) \frac{p(D \mid \boldsymbol{\theta}, H, I)}{p(D \mid H, I)},
$$

where $p(\boldsymbol{\theta} \mid H, I)$ denotes the prior probability distribution of our model parameters $\boldsymbol{\theta}$ given a model $H$ assuming the background information $I$. The quantity $p(D \mid \boldsymbol{\theta}, H, I)$ is the direct probability density (or likelihood function) of the data given the parameters, model and background information. The term $p(D \mid H, I)$ is known as the evidence of $D$ given our model and acts as a normalisation constant and does not affect the shape of the posterior distribution $p(\theta \mid D, H, I)[84]$. The background information $I$ (which represents our signal model, assumptions on Gaussian noise, physicality of parameters etc.) remains constant throughout our analysis and will not be mentioned hereafter.

\section{B. Likelihood}

When there is no signal in the data, we will say the null hypothesis $H_{\mathrm{n}}$, that the data contains any Gaussian noise, is true. 
Under these conditions, each $C$ value is drawn from a central $\chi_{4 M}^{2}$ distribution. Hence the $H_{\mathrm{n}}$ model is parametrized entirely by $M=2 m+1$, the number of sidebands in the template, where $m=\operatorname{ceil}[2 \pi f a]$ and depends on the search frequency $f$ and semi-major axis $a$.

The signal hypothesis $H_{\mathrm{GW}}$ is true if the data contains Gaussian noise plus a signal. The signal is defined by the set of parameters $\boldsymbol{\theta}=\left\{h_{0}, \cos \iota, \psi, \phi_{0}, a, P\right\}$. In the case of a signal present in the data, each $C$-statistic is drawn from a noncentral $\chi_{4 M}^{2}[\lambda(\theta)]$ distribution. The non-centrality parameter $\lambda(\boldsymbol{\theta})$ is defined by the signal parameters $\boldsymbol{\theta}$ and is given by

$$
\lambda(\boldsymbol{\theta})=\rho_{o}^{2} \sum_{n=-m}^{m} J_{n}^{2}\left(2 \pi f_{0} a_{0}\right)\left|\tilde{W}\left(f_{n}-f_{k}+l(n) \Delta f\right)\right|^{2} .
$$

It represents the total recovered optimal SNR contained within the sidebands. The likelihood function (the probability of our measured $C$ value given a parameter set $\boldsymbol{\theta}$ ) is then given by

$$
p(C \mid \boldsymbol{\theta})=\frac{1}{2} \exp \left(-\frac{1}{2}[C+\lambda(\boldsymbol{\theta})]\right)\left(\frac{C}{\lambda(\boldsymbol{\theta})}\right)^{M-\frac{1}{2}} \mathrm{I}_{2 M-1}(\sqrt{C \lambda(\boldsymbol{\theta})}) .
$$

It should be noted that although the quantity $M$ is a function of the semi-major axis and intrinsic gravitational wave frequency, it has been fixed according to the predefined number of teeth used in the sideband template. It is therefore not a function of $\boldsymbol{\theta}$.

\section{Priors}

When searching for weak signals, an overly prescriptive prior is undesirable because it may dominate the posterior. Hence, to be conservative, we adopt a uniform prior on $h_{0} \geq 0$; the possibility of $h_{0}=0$ excludes the use of a fully scaleinvariant Jeffreys prior $\propto 1 / h_{0}[81]$. The upper limit thus derived is consistent with the data, not just a re-iteration of the prior. The same $h_{0}$ prior has been adopted in previous searches [42, 53, 78, 85]; the motivation is discussed in more detail in [81].

Electromagnetic measurements of the orbital period $P$ and semi-major axis $a$ are assumed to carry normally distributed random errors. Hence we adopt Gaussian priors on the actual values $P_{0}$ and $a_{0}$. Specifically we take $p\left(P_{0}\right)=\mathcal{N}(P, \Delta P)$ and $p\left(a_{0}\right)=\mathcal{N}(a, \Delta a)$, where $\mathcal{N}(\mu, \sigma)$ denotes a Gaussian (normal) distribution with mean $\mu$ given by the electromagnetic observation and standard deviation $\sigma$ taken as the error in that observation.

The reference phase $\phi_{0}$ is automatically maximized over within the $\mathcal{F}$ stage of the analysis and therefore does not directly affect our (semi-coherent) analysis. The remaining amplitude parameters serve only to influence the optimal SNR, and therefore also the $C$. Without prior information from electromagnetic observations, we select the least informative (ignorant) physical priors such that $p(\cos \iota)=1 / 2$ and $p(\psi)=1 / 2 \pi$ on the domains $(-1,1)$ and $(0,2 \pi)$ respectively.

Any prior informative measurements (e.g. electromagnetic) on the amplitude parameters can be incorporated into the analysis, and serve to narrow the prior probability distributions.
For the Sco X-1 search, we can deduce measurements for $\cos \iota$ and $\psi$ from observations if we assume the rotation axes of the neutron star and accretion disk are aligned. This implies the neutron star inclination $\iota$ is equal to the orbital inclination. We can then set $\iota=44^{\circ} \pm 6^{\circ}$ from the inclination of the orbital plane suggested from observations of the radio components of Sco X-1 [74]. The same observations measure a position angle of these radio jets of $54 \pm 3^{\circ}$. Under the alignment assumption, the position angle is directly related to the gravitational wave polarization angle $\psi$, but with a phase shift of $180^{\circ}$, i.e. $\psi=234 \pm 3^{\circ}$. The above assumes the usual mass-quadrupole emission; for current-quadrupole emission from $r$-modes the results are the same with $\psi \rightarrow \psi+45^{\circ}$ [63].

\section{Posteriors}

The probability density function (PDF) on our search parameters given a single $C$-statistic value is

$$
p(\boldsymbol{\theta} \mid C) \propto p(C \mid \boldsymbol{\theta}) p(\boldsymbol{\theta}),
$$

and assuming that the prior PDFs on our parameters are independent, we can express the posterior PDF as

$$
p\left(h_{0}, \cos \iota, \psi, P, a \mid C\right) \propto p(C \mid \boldsymbol{\theta}) p\left(h_{0}\right) p(\cos \iota) p(\psi) p(P) p(a) .
$$

To perform inference on the gravitational wave strain $h_{0}$, we can marginalize this joint distribution over the other parameters leaving us with

$$
p\left(h_{0} \mid C\right) \propto \int_{-\infty}^{\infty} d a \int_{\infty}^{\infty} d P \int_{0}^{2 \pi} d \psi \int_{-1}^{1} d \cos \iota p(C \mid \boldsymbol{\theta}) \mathcal{N}(P, \Delta P) \mathcal{N}(a, \Delta a),
$$

where the flat priors on $h_{0}, \cos \iota$ and $\psi$ are absorbed into the proportionality. Note that the amplitude parameters act through the non-centrality parameter $\lambda(\theta)(E q .64)$ via the optimal SNR term (Eq.22), in the likelihood. The orbital parameters $a, P$ dictate the fraction of recovered SNR based on the mismatch in the predicted quantity and location of frequencymodulated sidebands (Eq. 64.

\section{E. Detection criteria and upper limits}

To determine whether or not a signal is present in the data, we compute a threshold value of the $C$-statistic such that the probability of achieving such a value or greater due to noise alone is $P_{\mathrm{a}}$, the false alarm probability. For a single measurement of the $C$-statistic this threshold is computed via

$$
\begin{aligned}
P_{\mathrm{a}} & =\int_{\mathcal{C}^{*}}^{\infty} p\left(C \mid h_{0}=0\right) \\
& =1-\mathcal{P}\left(2 M, C^{*} / 2\right),
\end{aligned}
$$


where the likelihood on $C$ in the noise only case becomes a central $\chi^{2}$ distribution and $\mathcal{P}(k / 2, x / 2)$ is the regularized Gamma function with $k$ degrees of freedom (the cumulative distribution function of a central $\chi_{k}^{2}$ distribution) defined at $x$.

In the case of $N$ measurements of the $C$-statistic, assuming statistically independent trials, the false alarm probability is given by

$$
\begin{aligned}
P_{a \mid N} & =1-\left(1-P_{\mathrm{a}}\right)^{N} \\
& =1-\left[\mathcal{P}\left(2 M, C^{*} / 2\right)\right]^{N} .
\end{aligned}
$$

The corresponding threshold $C_{N}^{*}$ such that the probability that one or more of these values exceeds that threshold is obtained by solving

$$
\mathcal{P}\left(2 M, C_{N}^{*} / 2\right)=\left(1-P_{a \mid N}\right)^{1 / N} .
$$

This solution is obtained numerically but can be represented notationally by

$$
C_{N}^{*}=2 \mathcal{P}^{-1}\left(2 M,\left[1-P_{a \mid N}\right]^{1 / N}\right),
$$

where $\mathcal{P}^{-1}$ represents the inverse function of $\mathcal{P}$.

In practice the $C$-statistic values will not be statistically independent as assumed above. The level of independence between adjacent frequency bins will be reduced (i.e. values will be become increasingly correlated) as the frequency resolution of the $C$-statistic is made finer. Additionally, due to the comb structure of the signal and template we find that results at frequencies separated by an integer number of frequencymodulated sideband spacings $j / P \mathrm{~Hz}$ for $j<m$ are highly correlated. This is due to the fact that these results will have been constructed from sums of $\mathcal{F}$-statistic values containing many common values. This latter effect is dominant over the former and as an approximation it can be assumed that within the frequency span of a single comb template there are $r T / P$ independent $C$-statistic results. ${ }^{4}$ The number of templates spans per unit search frequency is $\sim P / r M$ which leaves us with $\sim T / M$ independent $C$-statistic values per unit $\mathrm{Hz}$. This is a reduction by a factor of $M$ in the number of statistically independent results expected.

In the event of there being no candidate $C$-statistic values, the search allows us to compute upper-limits on the amplitude of gravitational waves from our target source. We define the upper limit on the wave strain $h_{0}$ as the value $h_{\mathrm{UL}}$ that bounds the fraction UL of the area of the marginalized posterior distribution $p\left(h_{0} \mid C\right)$. This value is obtained numerically by solving

$$
\mathrm{UL}=\int_{0}^{h_{\mathrm{UL}}} p\left(h_{0} \mid C\right) d h_{0} .
$$

We note that this procedure allows us to compute an upperlimit for each $C$-statistic value output from a search. The standard practice in continuous gravitational wave data analysis

\footnotetext{
${ }^{4}$ This comes from the number of bins in between each sideband, given by the sideband separation $1 / P$ divided by the bin size $d_{f}=\left(r T_{\mathrm{s}}\right)^{-1}$ (Eq. 57 .
}

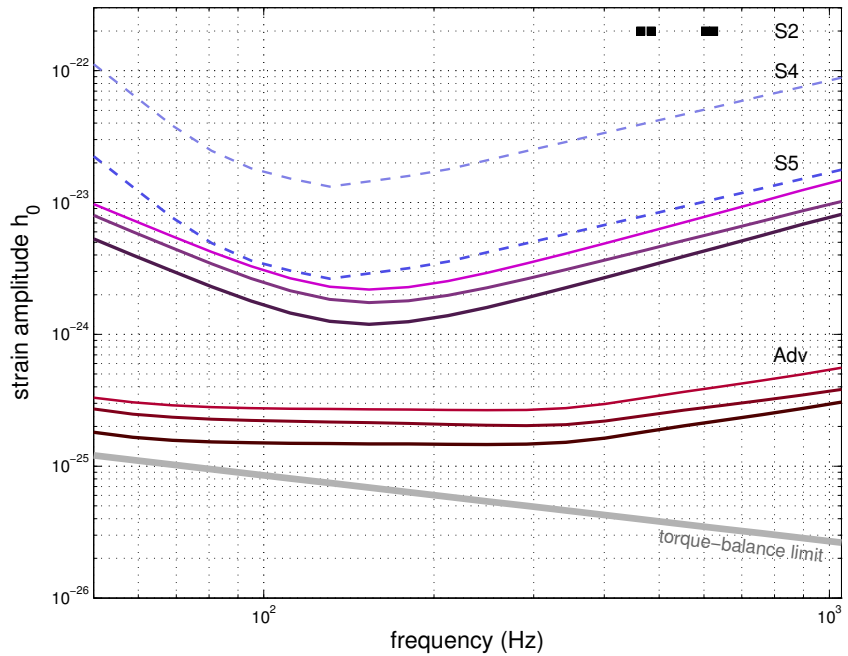

FIG. 5: Sensitivity estimate for a 10 day standard, approximate demodulated and approximate demodulated with known priors sideband search (fine, medium and bold solid curves respectively) using LIGO (H1L1) S5 data (upper, purple group), and using the 3-detector (H1L1V) advanced LIGO configuration (lower, red group). Also shown are results from the the previous coherent search for Sco X-1 in S2 data (solid black dashes) [53] and the maximum upper limits for each $\mathrm{Hz}$ band of the directed stochastic (radiometer) search in $\mathrm{S} 4$ and S5 data (light and dark blue dashed curves, respectively) [55, 56]. The theoretical torque-balance gravitational wave strain upper limit $\left(h_{0}^{\mathrm{EQ}}\right.$ from Eq. 7 for Sco X-1 is indicated by the thick gray straight line.

is to perform a frequentist upper-limit using computationally expensive Monte-Carlo simulations involving repeated signal injections. The results of these injections are then compared to loudest detection statistic recovered from the actual search [53]. In our approach, by virtue of the fact that we are able to compute upper-limits very efficiently for each $C$ statistic value and the upper-limit value is a monotonic function of $C$ we naturally also include the worst case (loudest event) result. The difference in the upper-limits obtained from both strategies then becomes an issue of Bayesian versus Frequentist interpretation. However, as shown in [83], in the limit of large SNR these upper-limit results become indistinguishable. When searching wide parameter spaces with large numbers of templates, as is the case for the sideband search, the most likely largest detection statistic value will be consistent with large SNR.

\section{SENSITIVITY}

The sensitivity of a future search can be predicted in a variety of ways. We choose to estimate the expected gravitational wave strain upper-limits for Initial LIGO data in order to compare against previous results. We also compare this to the expected sensitivity of the search with Advanced LIGO.

If the search is conducted such that the frequency space is split into small sub-bands, the sensitivity can be estimated by 
computing upper limits on the expected maximum from each of the sub-bands in Gaussian noise. This is equivalent to assigning a false alarm probability $P_{\mathrm{a}}=50 \%$ for $N=T_{\mathrm{s}} / M$ trials for each, say one $\mathrm{Hz}$ frequency sub-band, and using Eq.72 as the expected $C$-statistic. We can then calculate the posterior distribution of $h_{0}$ from Eqs. 65 and Eq 68 .

Figure 5 shows the sensitivity estimate of the $90 \%$ upper limit $(U L=0.9)$ for the sideband search in different modes: standard (described in Section III, represented by the thin solid curves), binary demodulated (described in Section III H. represented by the medium solid curves), and binary demodulated with known priors on $\cos \iota$ and $\psi$ (described in Section VIC, represented by the bold solid curves). It compares the sensitivity of the search in two-detector (H1L1) LIGO S5 data (upper, purple group) and three-detector (H1L1V) Advanced LIGO data (red group) with previous searches for Sco X-1 in LIGO S2 (black dashes) [53], S4 and S5 data (light and dark blue dashed curves, respectively) [55, 56]. The $h_{\mathrm{rms}}$ upper limit quoted in the latter two (radiometer) searches is optimized for the special case of a circularly polarized signal and hence less conservative than the angle averaged $h_{0}$ quoted in [53] and commonly used when quoting upper limits for continuous gravitational wave searches. Converting detectorstrain rms upper limits $h_{\text {rms }}$ to source-strain amplitude upper limit $h_{0}$ requires $h_{0} \sim 2.43 h_{\mathrm{rms}}$ (see [86]). The different confidence on the coherent S2 analysis and S4 ans S5 radiometer analyses (90 and 95\% respectively) also complexify any direct comparisons. The theoretical indirect wave strain limit $h_{0}^{\mathrm{EQ}}$ for gravitational waves from LMXBs represented by the thick gray line comes from Eq. 7.

The sensitivity curves in Fig. 5 show that the standard sideband search should improve current upper limits on gravitational waves from Sco X-1, even though it is limited to only 10 days of consecutive data. Running a demodulated search with known $\cos \iota$ and $\psi$ comes close to setting constraints on the indirect (torque-balance) upper limits in the advanced detector era.

\section{DISCUSSION}

We have described the sideband algorithm and shown that it provides a computationally efficient method to search for gravitational waves from sources in binary systems. It requires accurate knowledge of the sky position of the source and the orbital period of the binary, and less accurate knowledge on the semi-major axis. Effects of spin wandering can be ignored over a short enough coherent integration time.

The tolerance on the errors of relevant search parameters was computed, defining the range over which they can be assumed constant (Section IV). In light of these limits, electromagnetic observations suggest several candidates (Section V). Of these sources, Sco X-1 is identified as the strongest candi- date based on the gravitational wave strain recovered from the torque-balance argument (Eq. 7). In future, the search can also be directed at several other of the suitable LMXB candidates presented in Section $\mathrm{V}$.

A Bayesian upper limit strategy was presented in Section VI, rather than the frequentist methods commonly employed in frequency-based (LIGO) searches. Knowing the likelihood function in closed analytic form makes the Bayesian approach computationally more feasible than Monte Carlo simulations (see VIB). Knowing the gravitational wave polarization angle and inclination leads to additional sensitivity improvements using this framework (see VIC).

The sensitivity of the search, described in Section VII is estimated by performing the Bayesian analysis on the design curves of the S5 and Advanced LIGO noise floors. The sensitivity of a 10 day limited Sco X-1 directed sideband search compared to previous LIGO searches is shown in Fig. 5 . It shows that measurements of an orbital reference time and phase (the time and argument of periapse) can be employed to improve search sensitivity by a factor of 1.5 in the approximate demodulated version of the search. Also, prior information on the polarization and inclination of the gravitational wave signal constrains the upper limit calculation improving the sensitivity by another factor of 1.5 . In its most sensitive configuration (approximated binary demodulated assuming known $\cos \iota$ and $\psi$ in the Advanced detector era), the sideband search brings us closer to testing the theoretical indirect torque-balance limit.

The studies presented here assume pure Gaussian noise. The performance for realistic LIGO-like noise will be presented elsewhere, in a report on the results from the Sco X-1 directed search performed on LIGO (S5) data. The search could also look forward to running on the next-generation Advanced LIGO data.

\section{ACKNOWLEDGEMENTS}

This work has benefited from many useful discussions and helpful comments. In particular we would like to thank Karl Wette, Reinhard Prix, Evan Goetz, Holger Pletsch, Miroslav Shaltev, Badri Krishnan, Paul Lasky, Mark Bennett, Sterl Phinney and the LIGO Scientific Collaboration continuous waves working group. LS is indebted to Bruce Allen for his support and gratefully acknowledges the Max-Planck Society (Albert-Einstein Institut) for support and hospitality during periods when significant progress on this work was made. LS and CM acknowledge the Gravitational wave group at Cardiff University for their support. LS was supported by an Australian Postgraduate Award and a Melbourne University Overseas Research Experience Scholarship. AM and BJO acknowledge ARC grant DP-110103347. BJO was supported by NSF Grants PHY-0855589 and PHY-1206027.
[1] L. Bildsten, Astrophys. J. Lett. 501, L89 (1998), URL http: //stacks. iop.org/1538-4357/501/i=1/a=L89
[2] G. Ushomirsky, C. Cutler, and L. Bildsten, Mon. Not. R. As- 
tron. Soc. 319, 902 (2000), URL http://dx.doi.org/10. 1046/j.1365-8711.2000.03938.x

[3] B. J. Owen, Phys. Rev. Lett. 95, 211101 (2005), arXiv:astro$\mathrm{ph} / 0503399$.

[4] B. Haskell, N. Andersson, D. I. Jones, and L. Samuelsson, Phys. Rev. Lett. 99, 231101 (2007), URL http://link .aps .org/ doi/10.1103/PhysRevLett.99.231101

[5] L.-M. Lin, Phys. Rev. D76, 081502 (2007), 0708.2965.

[6] N. K. Johnson-McDaniel and B. J. Owen, Phys. Rev. D 88, 044004 (2013), 1208.5227.

[7] S. Bonazzola and E. Gourgoulhon, Astron. Astrophys. 312, 675 (1996), astro-ph/9602107.

[8] C. Cutler, Phys. Rev. D 66, 084025 (2002), arXiv:grqc/0206051.

[9] A. Melatos and D. J. B. Payne, Astrophys. J. 623, 1044 (2005), URL http://stacks.iop.org/0004-637X/623/ $i=2 / a=1044$

[10] M. Vigelius and A. Melatos, Mon. Not. R. Astron. Soc. 386, 1294 (2008), 0802.3238.

[11] B. Haskell, L. Samuelsson, K. Glampedakis, and N. Andersson, Mon. Not. R. Astron. Soc. 385, 531 (2008), 0705.1780.

[12] A. Mastrano and A. Melatos, Mon. Not. R. Astron. Soc. 421, 760 (2012), 1112.1542.

[13] D. I. Jones and N. Andersson, Mon. Not. R. Astron. Soc. 331, 203 (2002), arXiv:gr-qc/0106094.

[14] D. I. Jones, Mon. Not. R. Astron. Soc. 420, 2325 (2012), 1107.3503

[15] B. J. Owen, L. Lindblom, C. Cutler, B. F. Schutz, A. Vecchio, et al., Phys. Rev. D58, 084020 (1998), gr-qc/9804044.

[16] N. Andersson, K. D. Kokkotas, and N. Stergioulas, Astrophys. J. 516, 307 (1999), arXiv:astro-ph/9806089.

[17] R. Bondarescu, S. A. Teukolsky, and I. Wasserman, Phys. Rev. D76, 064019 (2007), 0704.0799.

[18] B. Haskell, N. Degenaar, and W. C. G. Ho, Mon. Not. R. Astron. Soc. 424, 93 (2012), 1201.2101.

[19] R. Bondarescu and I. Wasserman (2013), 1305.2335.

[20] M. Nayyar and B. J. Owen, Phys. Rev. D 73, 084001 (2006), URL http://link.aps.org/doi/10.1103/PhysRevD. 73.084001

[21] A. Melatos, Advances in Space Research 40, 1472 (2007), ISSN 0273-1177, URL http://www.sciencedirect.com/ science/article/pii/S0273117707003511

[22] C. A. van Eysden and A. Melatos, Class. Quant. Grav. 25, 225020 (2008), URL http://stacks.iop.org/ 0264-9381/25/i=22/a=225020

[23] M. Vigelius and A. Melatos, Mon. Not. R. Astron. Soc. 395, 1972 (2009), 0902.4264.

[24] N. Andersson, V. Ferrari, D. I. Jones, K. D. Kokkotas, B. Krishnan, J. S. Read, L. Rezzolla, and B. Zink, General Relativity and Gravitation 43, 409 (2011), 0912.0384.

[25] J. Papaloizou and J. E. Pringle, Mon. Not. R. Astron. Soc. 184, 501 (1978).

[26] R. V. Wagoner, Astrophys. J. 278, 345 (1984).

[27] F. Verbunt, Annu. Rev. Astron. Astrophys. 31, 93 (1993).

[28] A. L. Watts, B. Krishnan, L. Bildsten, and B. F. Schutz, Mon. Not. R. Astron. Soc. 389, 839 (2008).

[29] G. B. Cook, S. L. Shapiro, and S. A. Teukolsky, Astrophys. J. 424, 823 (1994).

[30] G. Ushomirsky, L. Bildsten, and C. Cutler, in American Institute of Physics Conference Series, edited by S. Meshkov (2000), vol. 523 of American Institute of Physics Conference Series, pp. 65-74, arXiv:astro-ph/0001129.

[31] D. Chakrabarty, E. H. Morgan, M. P. Muno, D. K. Galloway, R. Wijnands, M. van der Klis, and C. B. Markwardt, Nature
424, 42 (2003), arXiv:astro-ph/0307029.

[32] S. Bhattacharyya, Mon. Not. R. Astron. Soc. 377, 198 (2007), arXiv:astro-ph/0605510.

[33] D. K. Galloway, J. Lin, D. Chakrabarty, and J. M. Hartman, Astrophys. J. Lett. 711, L148 (2010), 0910.5546.

[34] B. P. Abbott, R. Abbott, R. Adhikari, P. Ajith, B. Allen, G. Allen, R. S. Amin, S. B. Anderson, W. G. Anderson, M. A. Arain, et al., Reports on Progress in Physics 72, 076901 (2009), 0711.3041

[35] J. Abadie, B. P. Abbott, R. Abbott, M. Abernathy, C. Adams, R. Adhikari, P. Ajith, B. Allen, G. Allen, E. Amador Ceron, et al., Nuclear Instruments and Methods in Physics Research A 624, 223 (2010), 1007.3973.

[36] URL http://www.ligo.caltech.edu/

[37] URL https://wwwcascina.virgo.infn.it/

[38] T. Accadia et al. (VIRGO Collaboration), JINST 7, P03012 (2012).

[39] H. Grote (LIGO Scientific Collaboration), Class. Quant. Grav. 27, 084003 (2010).

[40] URL http://www.geo600.org/

[41] P. R. Brady, T. Creighton, C. Cutler, and B. F. Schutz, Phys. Rev. D 57, 2101 (1998), URL http://link.aps.org/doi/ 10.1103/PhysRevD. 57.2101

[42] B. Abbott, R. Abbott, R. Adhikari, A. Ageev, B. Allen, R. Amin, S. B. Anderson, W. G. Anderson, M. Araya, H. Armandula, et al. (The LIGO Scientific Collaboration), Phys. Rev. D 69, 082004 (2004), arXiv:gr-qc/0308050.

[43] B. P. Abbott, R. Abbott, F. Acernese, R. Adhikari, P. Ajith, B. Allen, G. Allen, M. Alshourbagy, R. S. Amin, S. B. Anderson, et al. (The LIGO Scientific Collaboration and the Virgo Collaboration), Astrophys. J. 713, 671 (2010), 0909.3583.

[44] B. Abbott, R. Abbott, R. Adhikari, P. Ajith, B. Allen, G. Allen, R. Amin, S. B. Anderson, W. G. Anderson, M. A. Arain, et al. (The LIGO Scientific Collaboration), Astrophys. J. Lett. 683, L45 (2008), 0805.4758.

[45] J. Abadie, B. P. Abbott, R. Abbott, M. Abernathy, T. Accadia, F. Acernese, C. Adams, R. Adhikari, C. Affeldt, B. Allen, et al. (The LIGO Scientific Collaboration and the Virgo Collaboration), Astrophys. J. 737, 93 (2011), 1104.2712.

[46] J. Abadie, B. P. Abbott, R. Abbott, M. Abernathy, C. Adams, R. Adhikari, P. Ajith, B. Allen, G. Allen, E. Amador Ceron, et al. (LIGO Scientific Collaboration), Astrophys. J. 722, 1504 (2010), 1006.2535.

[47] B. Abbott, R. Abbott, R. Adhikari, J. Agresti, P. Ajith, B. Allen, R. Amin, S. B. Anderson, W. G. Anderson, M. Arain, et al. (LIGO Scientific Collaboration), Phys. Rev. D 77, 022001 (2008), 0708.3818.

[48] B. Abbott, R. Abbott, R. Adhikari, P. Ajith, B. Allen, G. Allen, R. Amin, D. P. Anderson, S. B. Anderson, W. G. Anderson, et al. (The LIGO Scientific Collaboration), Phys. Rev. D 79, 022001 (2009), 0804.1747.

[49] B. P. Abbott, R. Abbott, R. Adhikari, P. Ajith, B. Allen, G. Allen, R. S. Amin, S. B. Anderson, W. G. Anderson, M. A. Arain, et al. (The LIGO Scientific Collaboration), Phys. Rev. Lett. 102, 111102 (2009), 0810.0283.

[50] B. P. Abbott, R. Abbott, R. Adhikari, P. Ajith, B. Allen, G. Allen, R. S. Amin, S. B. Anderson, W. G. Anderson, M. A. Arain, et al. (The LIGO Scientific Collaboration and the Virgo Collaboration), Phys. Rev. D 80, 042003 (2009), 0905.1705.

[51] J. Abadie, B. P. Abbott, R. Abbott, T. D. Abbott, M. Abernathy, T. Accadia, F. Acernese, C. Adams, R. Adhikari, C. Affeldt, et al. (The LIGO Scientific Collaboration), Phys. Rev. D 85, 022001 (2012), 1110.0208.

[52] E. Goetz and K. Riles, Class. Quant. Grav. 28, 215006 
(2011), URL http://stacks.iop.org/0264-9381/28/i= $21 / a=215006$

[53] B. Abbott, R. Abbott, R. Adhikari, J. Agresti, P. Ajith, B. Allen, R. Amin, S. B. Anderson, W. G. Anderson, M. Arain, et al. (LIGO Scientific Collaboration), Phys. Rev. D 76, 082001 (2007).

[54] P. Jaranowski, A. Królak, and B. F. Schutz, Phys. Rev. D 58, 063001 (1998).

[55] B. Abbott, R. Abbott, R. Adhikari, J. Agresti, P. Ajith, B. Allen, R. Amin, S. B. Anderson, W. G. Anderson, M. Arain, et al. (LIGO Scientific Collaboration), Phys. Rev. D 76, 082003 (2007).

[56] J. Abadie, B. P. Abbott, R. Abbott, M. Abernathy, T. Accadia, F. Acernese, C. Adams, R. Adhikari, P. Ajith, B. Allen, et al. (LIGO Scientific Collaboration, Virgo Collaboration), Phys. Rev. Lett. 107, 271102 (2011), 1109.1809.

[57] P. R. Brady and T. Creighton, Phys. Rev. D 61, 082001 (2000), arXiv:gr-qc/9812014.

[58] R. Prix and M. Shaltev, Phys. Rev. D 85, 084010 (2012), 1201.4321.

[59] C. Messenger and G. Woan, Class. Quant. Grav. 24, 469 (2007), arXiv:gr-qc/0703155.

[60] S. M. Ransom, J. M. Cordes, and S. S. Eikenberry, Astrophys. J. 589, 911 (2003), URL http://stacks.iop.org/ $0004-637 \mathrm{X} / 589 / \mathrm{i}=2 / \mathrm{a}=911$

[61] T. M. Tauris and E. P. J. van den Heuvel (Cambridge University Press, 2006), chap. 16, URL http://dx.doi.org/10.1017/ CB09780511536281

[62] J. van Paradijs and N. White, ”Astrophys. J. Lett.” 447, L33+ (1995).

[63] B. J. Owen, Phys. Rev. D82, 104002 (2010), 1006.1994.

[64] J. H. Taylor and J. M. Weisberg, Astrophys. J. 345, 434 (1989).

[65] R. Prix, Phys. Rev. D 75, 023004 (2007).

[66] K.-H. Lo, F. K. Lamb, S. Boutloukos, and M. C. Miller, in AAS/High Energy Astrophysics Division (2011), vol. 12 of AAS/High Energy Astrophysics Division, p. 44.05.

[67] A. L. Watts, ”Ann. Rev. Astron. Astrophys.” 50, 609 (2012), 1203.2065 .

[68] M. van der Klis, Advances in Space Research 22, 925 (1998), ISSN 0273-1177, x-Ray Timing and Cosmic Gamma Ray Bursts, URL http://www.sciencedirect. com/science/article/B6V3S-3X8G9HQ-3/2/ f18d9fad8f3d9f9d15b6c69105c749b1.

[69] C. M. Zhang, Y. Y. Pan, J. Wang, A. Taani, and Y. H. Zhao, Int. J. Mod. Phys. Conference Series 12, 414 (2012).

[70] M. van der Klis, J. H. Swank, W. Zhang, K. Jahoda, E. H. Morgan, W. H. G. Lewin, B. Vaughan, and J. van Paradijs, Astrophys. J. Lett. 469, L1 (1996), URL http://stacks.iop. org $/ 1538-4357 / 469 / i=1 / a=L 1$

[71] C. F. Bradshaw, E. B. Fomalont, and B. J. Geldzahler, Astrophys. J. Lett. 512, L121 (1999), URL http://stacks.iop. org $/ 1538-4357 / 512 / i=2 / a=L 121$

[72] D. Steeghs and J. Casares, The Astrophysical Journal 568, 273 (2002), URL http://stacks.iop.org/0004-637X/ $568 / i=1 / a=273$

[73] D. Galloway, S. Premachandra, D. Steeghs, J. Casares, and C. Messenger, GWPAW Hannover.

[74] E. B. Fomalont, B. J. Geldzahler, and C. F. Bradshaw, The Astrophysical Journal 558, 283 (2001), URL http://stacks. iop.org/0004-637X/558/i=1/a=283.

[75] C. J. Messenger, Ph.D. thesis, The University of Birmingham (2006).

[76] H. V. Bradt, G. Moore, L. L. E. Braes, G. K. Miley, W. Forman, E. Kellogg, J. E. Hesser, W. E. Kunkel, W. A. Hiltner, R. Hjellming, et al., Astrophys. J. 197, 443 (1975).

[77] P. Hertz, B. Vaughan, K. S. Wood, J. P. Norris, K. Mitsuda, P. F. Michelson, and T. Dotani, Astrophys. J. 396, 201 (1992).

[78] B. Abbott, R. Abbott, R. Adhikari, J. Agresti, P. Ajith, B. Allen, R. Amin, S. B. Anderson, W. G. Anderson, M. Arain, et al. (The LIGO Scientific Collaboration), Phys. Rev. D 77, 022001 (2008), 0708.3818.

[79] J. Aasi, J. Abadie, B. P. Abbott, R. Abbott, T. D. Abbott, M. Abernathy, T. Accadia, F. Acernese, C. Adams, T. Adams, et al. (The LIGO Scientific Collaboration and the Virgo Collaboration), ArXiv e-prints (2012), 1207.7176.

[80] K. Wette, Phys. Rev. D 85, 042003 (2012), URL http: //link.aps.org/doi/10.1103/PhysRevD.85.042003

[81] R. J. Dupuis and G. Woan, Phys. Rev. D 72, 102002 (2005), arXiv:gr-qc/0508096.

[82] B. Abbott, R. Abbott, R. Adhikari, J. Agresti, P. Ajith, B. Allen, R. Amin, S. B. Anderson, W. G. Anderson, M. Arain, et al. (LIGO Scientific Collaboration and ALLEGRO Collaboration), Phys. Rev. D 76, 022001 (2007), arXiv:gr-qc/0703068.

[83] C. Röver, C. Messenger, and R. Prix, ArXiv e-prints (2011), 1103.2987.

[84] G. L. Bretthorst, Bayesian Spectrum Analysis and Parameter Estimation, vol. 48 of Lecture Notes in Statistics (SpringerVerlag, 1988).

[85] R. Prix and B. Krishnan, Class. Quant. Grav. 26, 204013 (2009), URL http://stacks.iop.org/0264-9381/26/i= $20 / a=204013$

[86] C. Messenger, Understanding the sensitivity of the stochastic radiometer analysis in terms of the strain tensor amplitude, LIGO Document T1000195 (2010). 